\title{
Experimental and Theoretical Radiative Lifetimes, Branching Fractions, Transition Probabilities, and Oscillator Strengths of Some Highly Excited Odd-parity Levels in Ir I
}

\author{
Lina Zhou ${ }^{1}$, Sébastien Gamrath ${ }^{2}$, Patrick Palmeri ${ }^{2}$, Pascal Quinet ${ }^{2,3}$, Meiqi Zhang ${ }^{1}$, and Zhenwen Dai ${ }^{1}$ (D \\ ${ }^{1}$ Key Laboratory of Physics and Technology for Advanced Batteries (Ministry of Education), College of Physics, Jilin University, Changchun 130012, People's \\ Republic of China; dai@jlu.edu.cn \\ ${ }^{2}$ Physique Atomique et Astrophysique, Université de Mons, B-7000 Mons, Belgium \\ ${ }^{3}$ IPNAS, Université de Liège, B-4000 Liège, Belgium \\ Received 2018 June 21; revised 2018 August 3; accepted 2018 August 6; published 2018 September 7
}

\begin{abstract}
Radiative lifetimes of 62 odd-parity levels of Ir I in the energy range between 32513.43 and $58625.10 \mathrm{~cm}^{-1}$ were measured using the time-resolved laser-induced fluorescence technique. The lifetime values obtained are in the range from 3.2 to $345 \mathrm{~ns}$. To our best knowledge, 59 results are reported for the first time. These are compared to computed data deduced from a pseudo-relativistic Hartree-Fock model including core-polarization contributions. From the combination of the experimental lifetime measurements and branching fraction calculations, a new set of transition probabilities and oscillator strengths is derived for 134 Ir I spectral lines of astrophysical interest in the wavelength region from 205 to $418 \mathrm{~nm}$.
\end{abstract}

Key words: atomic data - methods: data analysis - techniques: spectroscopic

\section{Introduction}

Atomic radiative parameters such as oscillator strengths and transition probabilities are very important in astrophysics, plasma diagnostics, analytical chemistry, etc. In astrophysics, the accuracy of stellar chemical abundances largely depends on the adequacy and accuracy of atomic radiative and structure data. For neutral iridium (Ir I, $Z=77$ ), there are two stable isotopes, ${ }^{191} \mathrm{Ir}$ and ${ }^{193} \mathrm{Ir}$, with relative abundances of $37.3 \%$ and $62.7 \%$ on the earth and a common nuclear spin of $3 / 2$. Its ionization limit was estimated to be $72323.9 \mathrm{~cm}^{-1}$ (Colarusso et al. 1997). Ir abundances in stars are of great significance not only in radioactive cosmochronology, but also in the structure and nucleosynthetic evolution of supernovae originating from the first stellar generation (Ivarsson et al. 2003).

Ramanujam \& Andersen (1978) reported the lifetimes of $\mathrm{z}^{6} \mathrm{D}_{9 / 2}^{\circ}$ and $\mathrm{z}^{6} \mathrm{~F}_{7 / 2,11 / 2}^{\circ}$ levels using the beam-sputtering technique. Gough et al. (1983) derived oscillator strengths for 27 transitions in Ir I by combination of the lifetimes of 25 oddparity levels obtained by the technique of laser-excited fluorescence from sputtered metal vapor and the branching fractions (BFs) by a hollow cathode lamp. Ivarsson et al. (2003) used the time-resolved laser-induced fluorescence (TRLIF) technique to measure the lifetime of $5 \mathrm{~d}^{7} 6 \mathrm{pz}^{6} \mathrm{~F}^{\circ} 11 / 2$ $\left(28452.32 \mathrm{~cm}^{-1}\right)$ and derived four transition probabilities for two levels by combination with BFs from Fourier transform spectroscopic measurement. Xu et al. (2007) measured lifetimes of nine odd-parity Ir I levels and four odd-parity Ir II levels using the TR-LIF method and performed corresponding calculations.

As far as we know, the experimental lifetimes of a total of 29 levels in the energy range $26307.49-41118.75 \mathrm{~cm}^{-1}$ in Ir I have been published so far. However, the lifetimes of high-lying levels above $42,000 \mathrm{~cm}^{-1}$ are still unknown. In the present work, we measured the lifetimes for 62 Ir I levels, including 56 levels above $42,000 \mathrm{~cm}^{-1}$, with the TR-LIF technique. These new data were combined with BFs obtained from pseudo-relativistic Hartree-Fock calculations, including core-polarization effects, to deduce semi-empirical transition probabilities and oscillator strengths for 134 Ir I spectral lines involving upper levels ranging from 32513.43 to $49823.54 \mathrm{~cm}^{-1}$.

\section{Experimental Setup}

The TR-LIF method for radiative lifetime measurements has been fully proven to be very reliable and has been used by many researchers over the past few years (see e.g., Ivarsson et al. 2004; Xu et al. 2007; Den Hartog et al. 2011). The experimental setup used for lifetime measurements in the present work is the same as that recently used by Tian et al. (2016), so only a brief description is given here.

A $532 \mathrm{~nm}$ Q-switched Nd:YAG laser with $8 \mathrm{~ns}$ pulse duration, $10 \mathrm{~Hz}$ repetition rate, and pulse energy of 5-10 mJ was used as an ablation beam to generate Ir laser plasma. A dye laser (Sirah Cobra-stretch) using DCM or Rhodamine 6G dyes, which was pumped by another Nd:YAG laser with the same performance parameters as the former, except for pulse energy, produced a tunable laser at $604-658 \mathrm{~nm}$ or $558-588 \mathrm{~nm}$. In order to achieve excitations of higher lying levels, this dye laser needed to be converted into the second or the third harmonic lights through one or two $\beta$-barium borate (BBO) type-I crystals, and sometimes the converted harmonic lights were extended as different orders of Stokes and anti-Stokes components by stimulated Raman scattering in a $\mathrm{H}_{2}$ gas cell. The excitation wavelength range for the levels studied in this paper is $204.404-381.832 \mathrm{~nm}$. The ablation laser was focused vertically on the Ir target in the vacuum chamber, where the excitation light passed horizontally through the Ir plasma at a distance of about $8 \mathrm{~mm}$ above the target to excite a level of interest. The laser-induced fluorescence from an excited level was collected by a fused silica lens into a grating monochromator and detected by a photomultiplier tube (PMT, Hamamatsu R3896). A $2.5 \mathrm{GHz}$ digital oscilloscope (Tektronix DPO7254) registered and averaged the transient signals from the PMT. A digital delay generator (SRS Model 535) was used to change the delay time between the excitation and ablation pulses. In this work, the lifetime measurements were performed at the delay times 


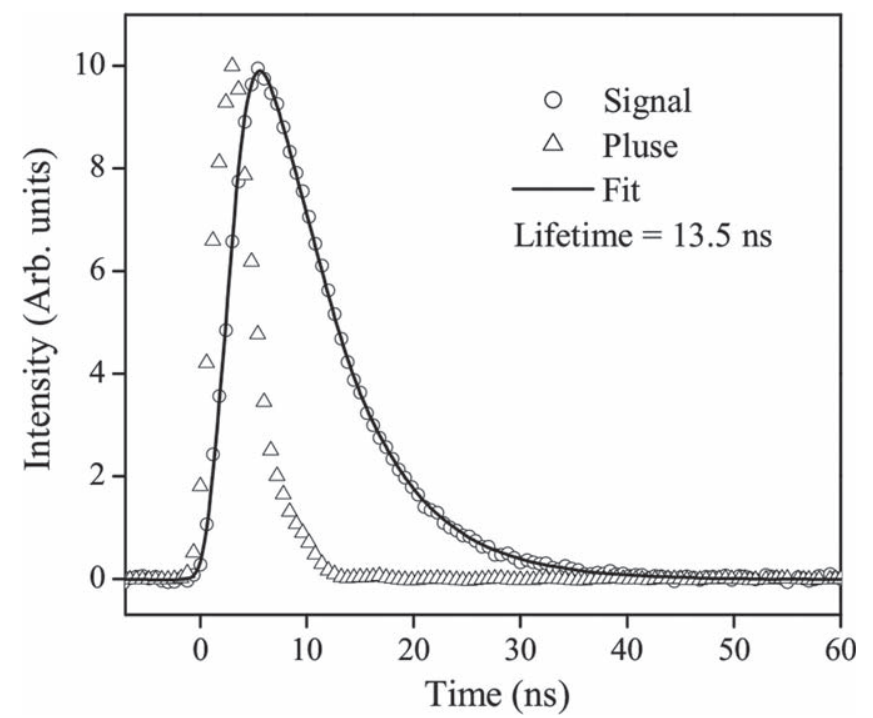

Figure 1. Convolution fitting to a typical fluorescence decay curve of the $52051.75 \mathrm{~cm}^{-1}$ level of Ir I from using a excitation pulse and an exponential function.

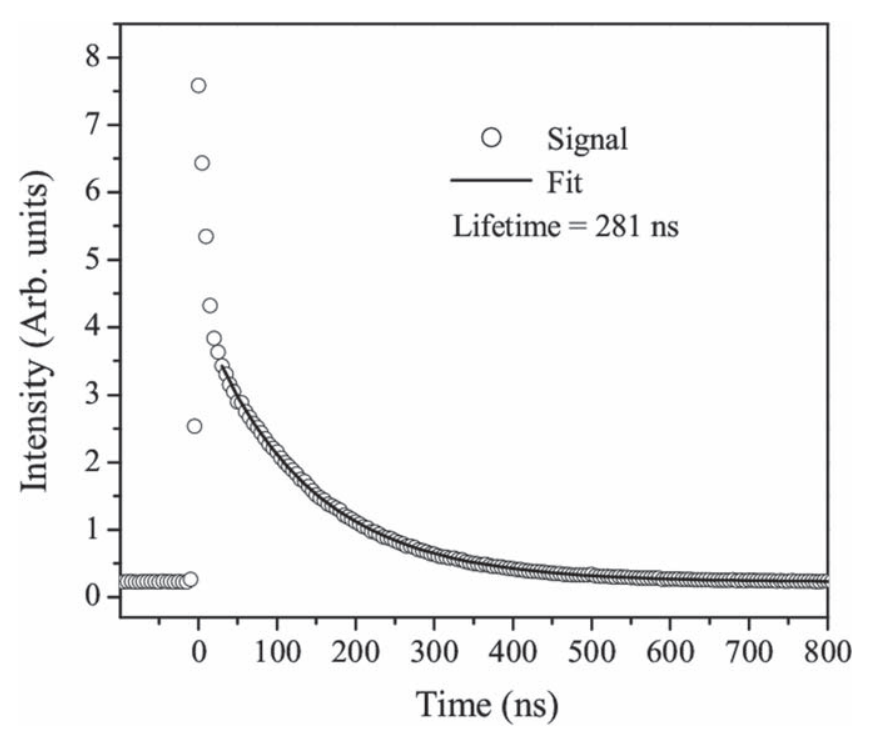

Figure 2. Exponential fitting to a typical fluorescence decay curve of the $35540.34 \mathrm{~cm}^{-1}$ level of Ir I.

from 4 to $45 \mu \mathrm{s}$. In order to wipe off Zeeman quantum beats induced by the Earth's magnetic field, a 100 G produced by a pair of Helmholtz coils in horizontal direction was employed to produce high-frequency Zeeman beats, which were out of the detection range of the PMT.

\section{Lifetime Measurements}

In the measurements, the single-step excitation scheme was employed. The target levels were populated from the ground state or some metastable states like those listed in Table 3. To ensure that only the studied level was excited, the excitation wavelength was carefully chosen from available excitation paths and monitored by a wavemeter (HighFinesse WS6). Moreover, the excited level under study was confirmed by

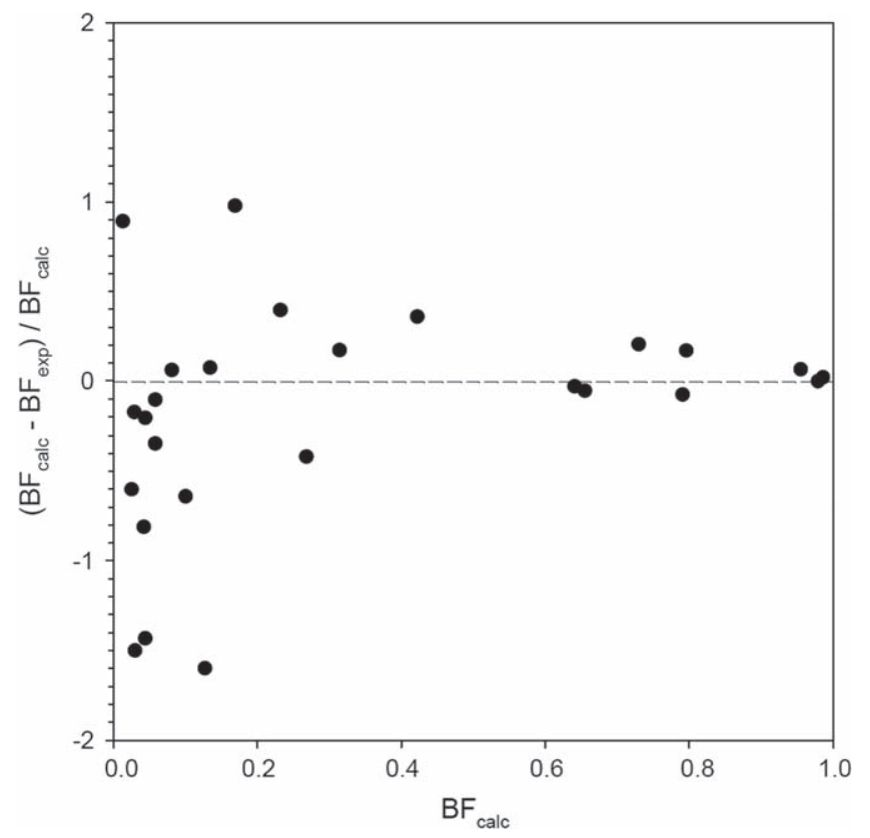

Figure 3. Comparison between the branching fractions calculated in the present work $\left(\mathrm{BF}_{\text {calc }}\right)$ using the $\mathrm{HFR}+\mathrm{CPOL}$ approach and the experimental values $\left(\mathrm{BF}_{\mathrm{exp}}\right)$ measured by Gough et al. (1983) and Ivarsson et al. (2003).

verifying that the observed fluorescence wavelengths were related to this level and their decay behaviors were the same.

All possible systematic effects influencing lifetime results were carefully checked, such as the radiation trapping, the collision deexcitation, and the flight-out-of-view effect. These effects can be eliminated by choosing appropriate experimental conditions through changing the excitation laser energy, the entrance slit of the monochromator and its position along the direction of atomic motion, and the delay time of the excitation pulse relative to the ablation one. More details on the elimination of systematic effects were described in our previous paper (Feng et al. 2011). In order to achieve a good signal-to-noise ratio in the time-resolved fluorescence decay curve, fluorescence signals induced by excitations of over 1000 pulses were acquired and averaged for each decay curve. For each level, more than 10 curves were registered under different experimental conditions, and the level's lifetime is the average of the lifetime values from these curves. Each curve gave two lifetime values. In an exponential fit, the two values were determined with two starting points of fit, respectively. One was a position with the intensity as strong as possible and out of the influence of stray light of the excitation laser, and the other was at half of the intensity of the former point. When using a convolutional fit, two lifetime values were evaluated by means of two excitation-pulse shapes recorded respectively before and after the fluorescence curve registration. The two lifetime values from a decay curve are in good agreement, and their mean value and its deviation from the two values were taken as the lifetime revealed by this curve and its systematic error, respectively. The uncertainty of the final lifetime result for a level is the standard deviations composited from the systematic errors and the statistical errors of different curve measurements.

From experience, when the lifetime value is shorter than 5 times the excitation-pulse width, a convolution fit to the 
Table 1

Calculated Energy Levels Compared to Experimentally Known Values for Even Parity in Ir I

\begin{tabular}{|c|c|c|c|c|}
\hline $\begin{array}{l}E_{\exp ^{\mathrm{a}}} \\
\left(\mathrm{cm}^{-1}\right)\end{array}$ & $\begin{array}{l}E_{\text {calc }}^{\mathrm{b}} \\
\left(\mathrm{cm}^{-1}\right)\end{array}$ & $\begin{array}{c}\Delta E \\
\left(\mathrm{~cm}^{-1}\right)\end{array}$ & $J$ & $\begin{array}{l}\text { LS Composition }{ }^{\mathrm{b}, \mathrm{c}} \\
(\%)\end{array}$ \\
\hline$\overline{0.00}$ & -51 & 51 & $9 / 2$ & $805 d^{7} 6 s^{24} F+135 d^{7} 6 s^{22} G$ \\
\hline 2834.98 & 2833 & 2 & $9 / 2$ & $945 d^{8}\left({ }^{3} F\right) 6 s{ }^{4} F$ \\
\hline 4078.94 & 4153 & -74 & $3 / 2$ & $325 \mathrm{~d}^{7} 6 \mathrm{~s}^{2}{ }^{2} \mathrm{P}+165 \mathrm{~d}^{8}\left({ }^{3} \mathrm{P}\right) 6 \mathrm{~s}^{2} \mathrm{P}+135 \mathrm{~d}^{8}\left({ }^{1} \mathrm{D}\right) 6 \mathrm{~s}^{2} \mathrm{D}$ \\
\hline 5784.62 & 5842 & -57 & $5 / 2$ & $235 \mathrm{~d}^{8}\left({ }^{1} \mathrm{D}\right) 6 \mathrm{~s}^{2} \mathrm{D}+215 \mathrm{~d}^{7} 6 \mathrm{~s}^{2}{ }^{4} \mathrm{~F}+85 \mathrm{~d}^{9}{ }^{2} \mathrm{D}$ \\
\hline 6323.91 & 6130 & 194 & $7 / 2$ & $545 \mathrm{~d}^{8}\left({ }^{3} \mathrm{~F}\right) 6 \mathrm{~s}{ }^{4} \mathrm{~F}+265 \mathrm{~d}^{8}\left({ }^{3} \mathrm{~F}\right) 6 \mathrm{~s}^{2} \mathrm{~F}$ \\
\hline 7106.61 & 7055 & 52 & $7 / 2$ & $795 \mathrm{~d}^{7} 6 \mathrm{~s}^{2}{ }^{4} \mathrm{~F}+115 \mathrm{~d}^{8}\left({ }^{3} \mathrm{~F}\right) 6 \mathrm{~s}{ }^{4} \mathrm{~F}$ \\
\hline 9877.54 & 9893 & -15 & $5 / 2$ & $325 d^{7} 6 s^{2}{ }^{4} F+245 d^{8}\left({ }^{3} F\right) 6 s{ }^{4} F+195 d^{8}\left({ }^{3} P\right) 6 s{ }^{4} P$ \\
\hline 10578.68 & 10697 & -118 & $3 / 2$ & $225 d^{8}\left({ }^{3} F\right) 6 s{ }^{4} F+225 d^{7} 6 s^{2}{ }^{4} P+195 d^{8}\left({ }^{1} D\right) 6 s{ }^{2} D$ \\
\hline 11831.09 & 11893 & -62 & $3 / 2$ & $535 \mathrm{~d}^{7} 6 \mathrm{~s}^{2}{ }^{4} \mathrm{~F}+195 \mathrm{~d}^{8}\left({ }^{3} \mathrm{~F}\right) 6 \mathrm{~s}{ }^{4} \mathrm{~F}+105 \mathrm{~d}^{8}\left({ }^{1} \mathrm{D}\right) 6 \mathrm{~s}^{2} \mathrm{D}$ \\
\hline 12218.47 & 12301 & -83 & $5 / 2$ & $585 \mathrm{~d}^{8}\left({ }^{3} \mathrm{~F}\right) 6 \mathrm{~s}{ }^{4} \mathrm{~F}+165 \mathrm{~d}^{8}\left({ }^{3} \mathrm{P}\right) 6 \mathrm{~s}{ }^{4} \mathrm{P}+125 \mathrm{~d}^{8}\left({ }^{3} \mathrm{~F}\right) 6 \mathrm{~s}^{2} \mathrm{~F}$ \\
\hline 12505.68 & 12467 & 39 & $1 / 2$ & $455 \mathrm{~d}^{7} 6 \mathrm{~s}^{2}{ }^{2} \mathrm{P}+285 \mathrm{~d}^{8}\left({ }^{3} \mathrm{P}\right) 6 \mathrm{~s}^{2} \mathrm{P}+225 \mathrm{~d}^{7} 6 \mathrm{~s}^{2}{ }^{4} \mathrm{P}$ \\
\hline 12951.67 & 13071 & -119 & $5 / 2$ & $695 d^{7} 6 s^{2}{ }^{4} P+175 d^{7} 6 s^{2}{ }^{4} F$ \\
\hline 13087.90 & 13132 & -44 & $7 / 2$ & $585 \mathrm{~d}^{8}\left({ }^{3} \mathrm{~F}\right) 6 \mathrm{~s}{ }^{2} \mathrm{~F}+325 \mathrm{~d}^{8}\left({ }^{3} \mathrm{~F}\right) 6 \mathrm{~s}{ }^{4} \mathrm{~F}$ \\
\hline 13939.80 & 13900 & 40 & $9 / 2$ & $545 \mathrm{~d}^{7} 6 \mathrm{~s}^{2}{ }^{2} \mathrm{G}+205 \mathrm{~d}^{7} 6 \mathrm{~s}^{2}{ }^{2} \mathrm{H}+135 \mathrm{~d}^{7} 6 \mathrm{~s}^{2}{ }^{4} \mathrm{~F}$ \\
\hline 16103.32 & 16098 & 5 & $5 / 2$ & $405 \mathrm{~d}^{8}\left({ }^{3} \mathrm{P}\right) 6 \mathrm{~s}{ }^{4} \mathrm{P}+5 \mathrm{~d}^{9}{ }^{2} \mathrm{D}+125 \mathrm{~d}^{8}\left({ }^{3} \mathrm{~F}\right) 6 \mathrm{~s}^{2} \mathrm{~F}$ \\
\hline 16565.35 & 16553 & 12 & $3 / 2$ & $605 \mathrm{~d}^{8}\left({ }^{3} \mathrm{P}\right) 6 \mathrm{~s}{ }^{4} \mathrm{P}+235 \mathrm{~d}^{8}\left({ }^{3} \mathrm{~F}\right) 6 \mathrm{~s}{ }^{4} \mathrm{~F}$ \\
\hline 16681.20 & 16693 & -12 & $1 / 2$ & $875 \mathrm{~d}^{8}\left({ }^{3} \mathrm{P}\right) 6 \mathrm{~s}{ }^{4} \mathrm{P}+85 \mathrm{~d}^{8}\left({ }^{1} \mathrm{~S}\right) 6 \mathrm{~s}^{2} \mathrm{~S}$ \\
\hline 17779.24 & 17772 & 7 & $7 / 2$ & $585 \mathrm{~d}^{7} 6 \mathrm{~s}^{2}{ }^{2} \mathrm{G}+295 \mathrm{~d}^{8}\left({ }^{1} \mathrm{G}\right) 6 \mathrm{~s}^{2} \mathrm{G}+65 \mathrm{~d}^{7} 6 \mathrm{~s}^{2}{ }^{2} \mathrm{~F}$ \\
\hline 18547.04 & 18539 & 8 & $3 / 2$ & $345 \mathrm{~d}^{7} 6 \mathrm{~s}^{2}{ }^{4} \mathrm{P}+295 \mathrm{~d}^{8}\left({ }^{3} \mathrm{P}\right) 6 \mathrm{~s}{ }^{4} \mathrm{P}+135 \mathrm{~d}^{8}\left({ }^{3} \mathrm{P}\right) 6 \mathrm{~s}{ }^{2} \mathrm{P}$ \\
\hline 19060.62 & 19149 & -88 & $5 / 2$ & $435 \mathrm{~d}^{8}\left({ }^{3} \mathrm{~F}\right) 6 \mathrm{~s}^{2} \mathrm{~F}+305 \mathrm{~d}^{92} \mathrm{D}+125 \mathrm{~d}^{7} 6 \mathrm{~s}^{2}{ }^{2} \mathrm{~F}$ \\
\hline 19593.25 & 19541 & 53 & $11 / 2$ & $955 \mathrm{~d}^{7} 6 \mathrm{~s}^{2}{ }^{2} \mathrm{H}$ \\
\hline 20236.70 & 20135 & 102 & $1 / 2$ & $685 \mathrm{~d}^{7} 6 \mathrm{~s}^{2}{ }^{4} \mathrm{P}+205 \mathrm{~d}^{8}\left({ }^{3} \mathrm{P}\right) 6 \mathrm{~s}^{2} \mathrm{P}$ \\
\hline 22110.24 & 22028 & 82 & $3 / 2$ & $255 \mathrm{~d}^{8}\left({ }^{1} \mathrm{D}\right) 6 \mathrm{~s}{ }^{2} \mathrm{D}+215 \mathrm{~d}^{9}{ }^{2} \mathrm{D}+185 \mathrm{~d}^{8}\left({ }^{3} \mathrm{~F}\right) 6 \mathrm{~s}{ }^{4} \mathrm{~F}$ \\
\hline 23310.36 & 23218 & 92 & $5 / 2$ & $385 \mathrm{~d}^{7} 6 \mathrm{~s}^{2}{ }^{2} \mathrm{D}+345 \mathrm{~d}^{9}{ }^{2} \mathrm{D}+75 \mathrm{~d}^{8}\left({ }^{1} \mathrm{D}\right) 6 \mathrm{~s}^{2} \mathrm{D}$ \\
\hline 23505.91 & 23509 & -3 & $9 / 2$ & $665 d^{8}\left({ }^{1} G\right) 6 s^{2} G+295 d^{7} 6 s^{2}{ }^{2} H$ \\
\hline 26229.48 & 26252 & -23 & $3 / 2$ & $435 d^{7} 6 s^{2}{ }^{2} D+265 d^{8}\left({ }^{3} P\right) 6 s{ }^{2} P+135 d^{7} 6 s^{2}{ }^{4} F$ \\
\hline 26365.16 & 26330 & 35 & $7 / 2$ & $635 d^{8}\left({ }^{1} G\right) 6 s^{2} G+255 d^{7} 6 s^{2}{ }^{2} G+65 d^{8}\left({ }^{3} F\right) 6 s^{2} F$ \\
\hline 26404.17 & 26293 & 111 & $5 / 2$ & $295 \mathrm{~d}^{8}\left({ }^{1} \mathrm{D}\right) 6 \mathrm{~s}^{2} \mathrm{D}+275 \mathrm{~d}^{7} 6 \mathrm{~s}^{2}{ }^{2} \mathrm{~F}+155 \mathrm{~d}^{7} 6 \mathrm{~s}^{2}{ }^{2} \mathrm{D}$ \\
\hline 27913.84 & 28076 & -162 & $9 / 2$ & $465 \mathrm{~d}^{7} 6 \mathrm{~s}^{2}{ }^{2} \mathrm{H}+285 \mathrm{~d}^{7} 6 \mathrm{~s}^{2}{ }^{2} \mathrm{G}+205 \mathrm{~d}^{8}\left({ }^{1} \mathrm{G}\right) 6 \mathrm{~s}^{2} \mathrm{G}$ \\
\hline 27970.05 & 27994 & -24 & $3 / 2$ & $565 \mathrm{~d}^{9}{ }^{2} \mathrm{D}+175 \mathrm{~d}^{8}\left({ }^{1} \mathrm{D}\right) 6 \mathrm{~s}^{2} \mathrm{D}+95 \mathrm{~d}^{7} 6 \mathrm{~s}^{2}{ }^{2} \mathrm{D}$ \\
\hline
\end{tabular}

Notes.

${ }^{a}$ From Kramida et al. (2018).

b This work.

${ }^{c}$ Only the first three eigenvector components are given, provided they are greater than or equal to $5 \%$.

fluorescence decay curve by combining the recorded excitation pulse with an exponential function is necessary for extracting more reliable lifetime value from the decay curve ( $\mathrm{Li}$ et al. 1999). A typical decay curve observed at $237.040 \mathrm{~nm}$ from the 52051.75 to $9877.54 \mathrm{~cm}^{-1}$ levels and fitted by convolution of a excitation-pulse shape and an exponential with $13.5 \mathrm{~ns}$ decay constant is shown in Figure 1. For the decay curves of longer lifetimes, exponential fits to the portions where the exciting pulse and its stray light ceased can give accurate decay constants. A typical decay curve observed at $351.594 \mathrm{~nm}$ from the 35540.34 to $7106.61 \mathrm{~cm}^{-1}$ levels fitted by an exponential function, giving rise to a lifetime value of $281 \mathrm{~ns}$, is shown in Figure 2. It can be seen from Figures 1 and 2 that the fit curves are in good agreement with the measured fluorescence curves, which benefit from the latter having a good signal-to-noise ratio. The lifetime results measured in the present work are shown in Table 3.

\section{Theoretical Calculations}

The theoretical approach used in the present work for computing the radiative parameters in Ir I was the pseudorelativistic Hartree-Fork (HFR) method of Cowan (1981) modified for taking core-polarization effects into account (HFR+CPOL), as described by Quinet et al. (1999, 2002). Since we considered the same model as the one used in our previous theoretical study of the iridium atom (Xu et al. 2007), just including more transitions in the calculations, only a brief summary is provided here. Twelve evenparity and nine odd-parity configurations were explicitly included in the calculations, namely $5 \mathrm{~d}^{7} 6 \mathrm{~s}^{2}, 5 \mathrm{~d}^{7} 6 \mathrm{p}^{2}, 5 \mathrm{~d}^{7} 6 \mathrm{~d}^{2}$, $5 d^{7} 6 s 7 s, 5 d^{7} 6 s 6 d, 5 d^{6} 6 s^{2} 7 s, 5 d^{6} 6 s^{2} 6 d, 5 d^{6} 6 s 6 p^{2}, 5 d^{8} 6 s$, $5 d^{8} 7 \mathrm{~s}, 5 d^{8} 6 \mathrm{~d}, 5 d^{9}$, and $5 d^{7} 6 s 6 p, 5 d^{7} 6 s 7 p, 5 d^{7} 6 s 5 f, 5 d^{7} 6 s 6 f$, $5 d^{6} 6 s^{2} 6 p, 5 d^{8} 6 p, 5 d^{8} 7 p, 5 d^{8} 5 f, 5 d^{8} 6 f$, respectively. The corepolarization effects were estimated using the dipole polarizability reported by Fraga et al. (1976) for an Ir IV ionic core, i.e., $\alpha_{\mathrm{d}}=6.48 a_{0}^{3}$, and a cutoff radius $r_{\mathrm{c}}=1.60 a_{0}$, corresponding to the expectation value of $\langle r\rangle$ for the outermost core orbital (5d), as obtained with the HFR method. Moreover, a semi-empirical fitting procedure was applied to the radial parameters related to the $5 d^{7} 6 s^{2}, 5 d^{8} 6 s$, $5 d^{9}, 5 d^{7} 6 s 6 p, 5 d^{6} 6 s^{2} 6 p$, and $5 d^{8} 6 p$ configurations. The details of this semi-empirical process can be found in $\mathrm{Xu}$ et al. (2007). It is important to note that the energy levels 
Table 2

Calculated Energy Levels Compared to Experimentally Known Values for Odd Parity in Ir I up to $50,000 \mathrm{~cm}^{-1}$

\begin{tabular}{|c|c|c|c|c|}
\hline $\begin{array}{l}E_{\exp ^{\mathrm{a}}} \\
\left(\mathrm{cm}^{-1}\right)\end{array}$ & $\begin{array}{c}E_{\text {calc }}^{\mathrm{b}} \\
\left(\mathrm{cm}^{-1}\right)\end{array}$ & $\begin{array}{c}\Delta E \\
\left(\mathrm{~cm}^{-1}\right)\end{array}$ & $J$ & $\begin{array}{l}\text { LS Composition }{ }^{\mathrm{b}, \mathrm{c}} \\
(\%)\end{array}$ \\
\hline 26307.50 & 26600 & -293 & $9 / 2$ & $525 d^{7}\left({ }^{4} F\right) 6 s 6 p{ }^{6} D+235 d^{7}\left({ }^{4} F\right) 6 s 6 p{ }^{6} F+85 d^{7}\left({ }^{2} G\right) 6 s 6 p{ }^{4} F$ \\
\hline 28452.32 & 28290 & 162 & $11 / 2$ & $635 d^{7}\left({ }^{4} F\right) 6 s 6 p{ }^{6} F+195 d^{7}\left({ }^{4} F\right) 6 s 6 p{ }^{6} G+75 d^{7}\left({ }^{4} F\right) 6 s 6 p{ }^{4} G$ \\
\hline 30529.66 & 30703 & -173 & $7 / 2$ & $375 d^{7}\left({ }^{4} F\right) 6 s 6 p{ }^{6} D+285 d^{7}\left({ }^{4} F\right) 6 s 6 p{ }^{6} F+65 d^{7}\left({ }^{2} G\right) 6 s 6 p{ }^{4} F$ \\
\hline 32463.58 & 32524 & -60 & $3 / 2$ & $245 \mathrm{~d}^{7}\left({ }^{2} \mathrm{P}\right) 6 \mathrm{~s} 6 \mathrm{p}{ }^{4} \mathrm{P}+95 \mathrm{~d}^{8}\left({ }^{3} \mathrm{P}\right) 6 \mathrm{p}{ }^{4} \mathrm{P}+85 \mathrm{~d}^{7}\left({ }^{4} \mathrm{P}\right) 6 \mathrm{~s} 6 \mathrm{p}{ }^{6} \mathrm{D}$ \\
\hline 32513.43 & 32372 & 141 & $9 / 2$ & $275 d^{7}\left({ }^{4} F\right) 6 s 6 p{ }^{6} G+235 d^{7}\left({ }^{4} F\right) 6 s 6 p{ }^{6} F+185 d^{7}\left({ }^{4} F\right) 6 s 6 p{ }^{6} D$ \\
\hline 32830.78 & 32892 & -61 & $13 / 2$ & $885 \mathrm{~d}^{7}\left({ }^{4} \mathrm{~F}\right) 6 \mathrm{~s} 6 \mathrm{p}{ }^{6} \mathrm{G}+115 \mathrm{~d}^{7}\left({ }^{2} \mathrm{G}\right) 6 \mathrm{~s} 6 \mathrm{p}{ }^{4} \mathrm{H}$ \\
\hline 33064.83 & 32969 & 96 & $5 / 2$ & $165 \mathrm{~d}^{7}\left({ }^{2} \mathrm{P}\right) 6 \mathrm{~s} 6 \mathrm{p}{ }^{4} \mathrm{P}+135 \mathrm{~d}^{7}\left({ }^{4} \mathrm{P}\right) 6 \mathrm{~s} 6 \mathrm{p}{ }^{6} \mathrm{~S}+115 \mathrm{~d}^{7}\left({ }^{2} \mathrm{P}\right) 6 \mathrm{~s} 6 \mathrm{p}{ }^{4} \mathrm{D}$ \\
\hline 33874.43 & 33960 & -86 & $7 / 2$ & $385 d^{8}\left({ }^{3} F\right) 6 p{ }^{4} D+95 d^{7}\left({ }^{4} F\right) 6 s 6 p{ }^{4} D+85 d^{7}\left({ }^{4} F\right) 6 s 6 p{ }^{6} F$ \\
\hline 34180.48 & 34063 & 117 & $11 / 2$ & $285 d^{7}\left({ }^{4} F\right) 6 s 6 p{ }^{6} G+255 d^{7}\left({ }^{4} F\right) 6 s 6 p{ }^{6} F+235 d^{7}\left({ }^{4} F\right) 6 s 6 p{ }^{4} G$ \\
\hline 34919.83 & 34959 & -39 & $5 / 2$ & $455 \mathrm{~d}^{7}\left({ }^{4} \mathrm{~F}\right) 6 \mathrm{~s} 6 \mathrm{p}{ }^{6} \mathrm{~F}+125 \mathrm{~d}^{7}\left({ }^{4} \mathrm{~F}\right) 6 \mathrm{~s} 6 \mathrm{p}{ }^{6} \mathrm{D}$ \\
\hline 35080.80 & 35031 & 50 & $9 / 2$ & $165 d^{8}\left({ }^{3} F\right) 6 p{ }^{4} F+145 d^{7}\left({ }^{4} F\right) 6 s 6 p{ }^{4} F+95 d^{7}\left({ }^{4} F\right) 6 s 6 p{ }^{6} F$ \\
\hline 35410.63 & 35191 & 220 & $7 / 2$ & $215 d^{7}\left({ }^{4} F\right) 6 s 6 p{ }^{6} \mathrm{G}+145 \mathrm{~d}^{7}\left({ }^{4} \mathrm{~F}\right) 6 s 6 \mathrm{p}{ }^{6} \mathrm{~F}+115 \mathrm{~d}^{7}\left({ }^{4} \mathrm{~F}\right) 6 \mathrm{~s} 6 \mathrm{p}{ }^{6} \mathrm{D}$ \\
\hline 35540.34 & 35600 & -60 & $5 / 2$ & $235 d^{7}\left({ }^{4} P\right) 6 s 6 p{ }^{6} S+155 d^{7}\left({ }^{4} F\right) 6 s 6 p{ }^{6} D+105 d^{7}\left({ }^{4} P\right) 6 s 6 p{ }^{6} D$ \\
\hline 35647.94 & 35629 & 19 & $1 / 2$ & $205 \mathrm{~d}^{7}\left({ }^{2} \mathrm{P}\right) 6 \mathrm{~s} 6 \mathrm{p}{ }^{4} \mathrm{P}+205 \mathrm{~d}^{7}\left({ }^{4} \mathrm{~F}\right) 6 \mathrm{~s} 6 \mathrm{p}{ }^{6} \mathrm{~F}+105 \mathrm{~d}^{7}\left({ }^{2} \mathrm{D}\right) 6 \mathrm{~s} 6 \mathrm{p}{ }^{4} \mathrm{D}$ \\
\hline 36390.15 & 36436 & -46 & $3 / 2$ & $515 \mathrm{~d}^{7}\left({ }^{4} \mathrm{~F}\right) 6 \mathrm{~s} 6 \mathrm{p}{ }^{6} \mathrm{~F}+75 \mathrm{~d}^{7}\left({ }^{2} \mathrm{D}\right) 6 \mathrm{~s} 6 \mathrm{p}{ }^{4} \mathrm{D}+55 \mathrm{~d}^{7}\left({ }^{4} \mathrm{~F}\right) 6 \mathrm{~s} 6 \mathrm{p}{ }^{6} \mathrm{D}$ \\
\hline 37446.13 & 37408 & 38 & $5 / 2$ & $385 d^{7}\left({ }^{4} F\right) 6 s 6 p{ }^{6} G+195 d^{7}\left({ }^{4} P\right) 6 s 6 p{ }^{6} S+95 d^{7}\left({ }^{4} F\right) 6 s 6 p{ }^{6} D$ \\
\hline 37515.31 & 37516 & -1 & $7 / 2$ & $175 d^{7}\left({ }^{4} F\right) 6 s 6 p{ }^{6} G+155 d^{7}\left({ }^{2} P\right) 6 s 6 p{ }^{4} D+125 d^{7}\left({ }^{4} F\right) 6 s 6 p{ }^{4} D$ \\
\hline 37692.75 & 37580 & 113 & $3 / 2$ & $255 \mathrm{~d}^{7}\left({ }^{4} \mathrm{~F}\right) 6 \mathrm{~s} 6 \mathrm{p}{ }^{6} \mathrm{G}+135 \mathrm{~d}^{7}\left({ }^{2} \mathrm{P}\right) 6 \mathrm{~s} 6 \mathrm{p}{ }^{4} \mathrm{D}+125 \mathrm{~d}^{7}\left({ }^{2} \mathrm{D}\right) 6 \mathrm{~s} 6 \mathrm{p}{ }^{4} \mathrm{~F}$ \\
\hline 37871.69 & 37838 & 34 & $9 / 2$ & $215 d^{8}\left({ }^{3} F\right) 6 p{ }^{4} F+215 d^{7}\left({ }^{4} F\right) 6 s 6 p{ }^{4} F+185 d^{7}\left({ }^{4} F\right) 6 s 6 p{ }^{6} F$ \\
\hline 38120.94 & 38082 & 39 & $1 / 2$ & $355 \mathrm{~d}^{7}\left({ }^{4} \mathrm{~F}\right) 6 \mathrm{~s} 6 \mathrm{p}{ }^{6} \mathrm{~F}+85 \mathrm{~d}^{7}\left({ }^{2} \mathrm{P}\right) 6 \mathrm{~s} 6 \mathrm{p}{ }^{4} \mathrm{P}+75 \mathrm{~d}^{7}\left({ }^{4} \mathrm{P}\right) 6 \mathrm{~s} 6 \mathrm{p}{ }^{6} \mathrm{D}$ \\
\hline 38158.24 & 38182 & -24 & $7 / 2$ & $175 d^{7}\left({ }^{4} F\right) 6 s 6 p{ }^{6} G+145 d^{7}\left({ }^{4} F\right) 6 s 6 p{ }^{4} F+95 d^{6}\left({ }^{5} D\right) 6 s^{2} 6 p{ }^{6} D$ \\
\hline 38229.75 & 38255 & -25 & $9 / 2$ & $295 \mathrm{~d}^{8}\left({ }^{3} \mathrm{~F}\right) 6 \mathrm{p}{ }^{2} \mathrm{G}+195 \mathrm{~d}^{8}\left({ }^{3} \mathrm{~F}\right) 6 \mathrm{p}{ }^{4} \mathrm{G}+155 \mathrm{~d}^{7}\left({ }^{4} \mathrm{~F}\right) 6 \mathrm{~s} 6 \mathrm{p}{ }^{6} \mathrm{G}$ \\
\hline 38358.13 & 38370 & -12 & $5 / 2$ & $225 d^{7}\left({ }^{4} \mathrm{P}\right) 6 \mathrm{~s} 6 \mathrm{p}{ }^{6} \mathrm{~S}+105 \mathrm{~d}^{8}\left({ }^{3} \mathrm{~F}\right) 6 \mathrm{p}{ }^{4} \mathrm{D}+105 \mathrm{~d}^{7}\left({ }^{4} \mathrm{~F}\right) 6 \mathrm{~s} 6 \mathrm{p}{ }^{6} \mathrm{G}$ \\
\hline 38484.74 & 38582 & -97 & $3 / 2$ & $325 d^{7}\left({ }^{4} F\right) 6 s 6 p{ }^{6} D+185 d^{7}\left({ }^{4} P\right) 6 s 6 p{ }^{6} D+165 d^{7}\left({ }^{4} F\right) 6 s 6 p{ }^{6} G$ \\
\hline 38568.05 & 38537 & 31 & $7 / 2$ & $135 d^{7}\left({ }^{4} F\right) 6 s 6 p{ }^{4} D+105 d^{7}\left({ }^{4} F\right) 6 s 6 p{ }^{6} D+95 d^{7}\left({ }^{4} F\right) 6 s 6 p{ }^{4} F$ \\
\hline 39289.28 & 39384 & -95 & $1 / 2$ & $155 d^{7}\left({ }^{4} F\right) 6 s 6 p{ }^{6} \mathrm{D}+155 \mathrm{~d}^{7}\left({ }^{4} \mathrm{P}\right) 6 \mathrm{~s} 6 \mathrm{p}{ }^{6} \mathrm{D}+105 \mathrm{~d}^{8}\left({ }^{1} \mathrm{D}\right) 6 \mathrm{p}^{2} \mathrm{P}$ \\
\hline 39324.57 & 39312 & 13 & $5 / 2$ & $115 \mathrm{~d}^{7}\left({ }^{4} \mathrm{~F}\right) 6 \mathrm{~s} 6 \mathrm{p}{ }^{6} \mathrm{D}+95 \mathrm{~d}^{8}\left({ }^{1} \mathrm{D}\right) 6 \mathrm{p}{ }^{2} \mathrm{~F}+85 \mathrm{~d}^{7}\left({ }^{4} \mathrm{P}\right) 6 \mathrm{~s} 6 \mathrm{p}{ }^{6} \mathrm{D}$ \\
\hline 39805.97 & 39864 & -58 & $5 / 2$ & $165 \mathrm{~d}^{8}\left({ }^{3} \mathrm{~F}\right) 6 \mathrm{p}{ }^{4} \mathrm{D}+85 \mathrm{~d}^{7}\left({ }^{2} \mathrm{P}\right) 6 \mathrm{~s} 6 \mathrm{p}{ }^{4} \mathrm{P}+55 \mathrm{~d}^{7}\left({ }^{4} \mathrm{~F}\right) 6 \mathrm{~s} 6 \mathrm{p}{ }^{6} \mathrm{~F}$ \\
\hline 39940.27 & 39821 & 119 & $11 / 2$ & $365 d^{8}\left({ }^{3} F\right) 6 p{ }^{4} G+295 d^{7}\left({ }^{4} F\right) 6 s 6 p{ }^{6} G+185 d^{7}\left({ }^{4} F\right) 6 s 6 p{ }^{4} G$ \\
\hline 40291.19 & 40343 & -52 & $7 / 2$ & $155 \mathrm{~d}^{8}\left({ }^{3} \mathrm{~F}\right) 6 \mathrm{p}{ }^{2} \mathrm{~F}+135 \mathrm{~d}^{8}\left({ }^{3} \mathrm{~F}\right) 6 \mathrm{p}{ }^{4} \mathrm{~F}$ \\
\hline 40389.83 & 40270 & 120 & $9 / 2$ & $225 d^{7}\left({ }^{4} F\right) 6 s 6 p{ }^{6} G+185 d^{7}\left({ }^{4} F\right) 6 s 6 p{ }^{4} F$ \\
\hline 40524.73 & 40640 & -115 & $3 / 2$ & $235 d^{7}\left({ }^{4} F\right) 6 s 6 p{ }^{6} G+115 d^{7}\left({ }^{2} P\right) 6 s 6 p{ }^{4} D+95 d^{6}\left({ }^{5} D\right) 6 s^{2} 6 p{ }^{6} D$ \\
\hline 40710.78 & 40766 & -55 & $7 / 2$ & $275 d^{7}\left({ }^{4} P\right) 6 s 6 p{ }^{6} D+145 d^{6}\left({ }^{5} D\right) 6 s^{2} 6 p{ }^{6} D$ \\
\hline 41118.71 & 41076 & 43 & $9 / 2$ & $275 d^{6}\left({ }^{5} D\right) 6 s^{2} 6 p{ }^{6} D+255 d^{7}\left({ }^{4} P\right) 6 s 6 p{ }^{6} D+65 d^{7}\left({ }^{4} F\right) 6 s 6 p{ }^{4} F$ \\
\hline 41210.33 & 41217 & -7 & $1 / 2$ & $325 d^{7}\left({ }^{4} F\right) 6 s 6 p{ }^{6} D+215 d^{6}\left({ }^{5} D\right) 6 s^{2} 6 p{ }^{6} D+85 d^{7}\left({ }^{2} P\right) 6 s 6 p{ }^{4} D$ \\
\hline 41522.22 & 41640 & -118 & $5 / 2$ & $175 \mathrm{~d}^{7}\left({ }^{4} \mathrm{P}\right) 6 \mathrm{~s} 6 \mathrm{p}{ }^{6} \mathrm{D}+115 \mathrm{~d}^{6}\left({ }^{5} \mathrm{D}\right) 6 \mathrm{~s}^{2} 6 \mathrm{p}{ }^{6} \mathrm{D}+85 \mathrm{~d}^{8}\left({ }^{3} \mathrm{~F}\right) 6 \mathrm{p}{ }^{4} \mathrm{D}$ \\
\hline 42014.44 & 42099 & -85 & $1 / 2$ & $295 \mathrm{~d}^{7}\left({ }^{2} \mathrm{P}\right) 6 \mathrm{~s} 6 \mathrm{p}{ }^{4} \mathrm{D}+165 \mathrm{~d}^{8}\left({ }^{3} \mathrm{P}\right) 6 \mathrm{p}{ }^{4} \mathrm{D}+95 \mathrm{~d}^{7}\left({ }^{2} \mathrm{P}\right) 6 \mathrm{~s} 6 \mathrm{p}{ }^{4} \mathrm{P}$ \\
\hline 42029.14 & 41896 & 133 & $3 / 2$ & $145 \mathrm{~d}^{8}\left({ }^{1} \mathrm{D}\right) 6 \mathrm{p}^{2} \mathrm{D}+95 \mathrm{~d}^{7}\left({ }^{4} \mathrm{~F}\right) 6 \mathrm{~s} 6 \mathrm{p}{ }^{6} \mathrm{~F}+75 \mathrm{~d}^{7}\left({ }^{2} \mathrm{D}\right) 6 \mathrm{~s} 6 \mathrm{p}^{2} \mathrm{D}$ \\
\hline 42131.82 & 42279 & -147 & $11 / 2$ & $365 d^{8}\left({ }^{3} F\right) 6 p{ }^{4} G+205 d^{7}\left({ }^{4} F\right) 6 s 6 p{ }^{4} G$ \\
\hline 42267.86 & 42382 & -114 & $5 / 2$ & $145 d^{6}\left({ }^{5} D\right) 6 s^{2} 6 p{ }^{6} D+115 d^{7}\left({ }^{4} F\right) 6 s 6 p{ }^{4} F+75 d^{7}\left({ }^{4} P\right) 6 s 6 p{ }^{6} D$ \\
\hline 42279.28 & 42068 & 211 & $9 / 2$ & $155 d^{6}\left({ }^{5} \mathrm{D}\right) 6 \mathrm{~s}^{2} 6 \mathrm{p}^{6} \mathrm{D}+145 \mathrm{~d}^{7}\left({ }^{4} \mathrm{~F}\right) 6 \mathrm{~s} 6 \mathrm{p}{ }^{6} \mathrm{~F}$ \\
\hline 43071.78 & 43021 & 51 & $5 / 2$ & $85 d^{8}\left({ }^{3} F\right) 6 p{ }^{4} G+85 d^{8}\left({ }^{3} F\right) 6 p{ }^{4} F+65 d^{8}\left({ }^{3} F\right) 6 p{ }^{4} D$ \\
\hline 43176.15 & 42973 & 203 & $7 / 2$ & $205 \mathrm{~d}^{8}\left({ }^{3} \mathrm{~F}\right) 6 \mathrm{p}{ }^{4} \mathrm{G}+85 \mathrm{~d}^{7}\left({ }^{2} \mathrm{~F}\right) 6 \mathrm{~s} 6 \mathrm{p}{ }^{4} \mathrm{G}+75 \mathrm{~d}^{7}\left({ }^{4} \mathrm{~F}\right) 6 \mathrm{~s} 6 \mathrm{p}{ }^{4} \mathrm{G}$ \\
\hline 43200.89 & 43160 & 41 & $3 / 2$ & $135 d^{8}\left({ }^{3} F\right) 6 p{ }^{4} D+125 d^{7}\left({ }^{4} F\right) 6 s 6 p{ }^{4} D+105 d^{7}\left({ }^{4} F\right) 6 s 6 p{ }^{4} F$ \\
\hline 43592.21 & 43487 & 105 & $7 / 2$ & $195 d^{7}\left({ }^{4} P\right) 6 s 6 p{ }^{6} P+175 d^{6}\left({ }^{5} D\right) 6 s^{2} 6 p{ }^{6} P+145 d^{7}\left({ }^{4} F\right) 6 s 6 p{ }^{6} D$ \\
\hline 44569.85 & 44474 & 96 & $3 / 2$ & $125 d^{7}\left({ }^{4} \mathrm{P}\right) 6 \mathrm{~s} 6 \mathrm{p}{ }^{6} \mathrm{D}+85 \mathrm{~d}^{7}\left({ }^{4} \mathrm{P}\right) 6 \mathrm{~s} 6 \mathrm{p}{ }^{4} \mathrm{~S}$ \\
\hline 44596.77 & 44567 & 30 & $5 / 2$ & $145 \mathrm{~d}^{7}\left({ }^{4} \mathrm{P}\right) 6 \mathrm{~s} 6 \mathrm{p}{ }^{6} \mathrm{P}+125 \mathrm{~d}^{8}\left({ }^{3} \mathrm{~F}\right) 6 \mathrm{p}{ }^{4} \mathrm{~F}+85 \mathrm{~d}^{7}\left({ }^{2} \mathrm{P}\right) 6 \mathrm{~s} 6 \mathrm{p}{ }^{4} \mathrm{D}$ \\
\hline 44642.67 & 44883 & -240 & $7 / 2$ & $155 d^{7}\left({ }^{4} F\right) 6 s 6 p{ }^{4} D+135 d^{6}\left({ }^{5} D\right) 6 s^{2} 6 p{ }^{6} D+105 d^{7}\left({ }^{2} G\right) 6 s 6 p{ }^{2} G$ \\
\hline 44652.43 & 44734 & -82 & $9 / 2$ & $215 d^{7}\left({ }^{4} F\right) 6 s 6 p{ }^{4} G+85 d^{7}\left({ }^{2} G\right) 6 s 6 p{ }^{2} H+75 d^{7}\left({ }^{4} P\right) 6 s 6 p{ }^{6} D$ \\
\hline 44785.44 & 44782 & 3 & $3 / 2$ & $165 d^{7}\left({ }^{4} P\right) 6 s 6 p{ }^{6} D+95 d^{6}\left({ }^{5} D\right) 6 s^{2} 6 p{ }^{6} D+85 d^{7}\left({ }^{4} P\right) 6 s 6 p{ }^{6} P$ \\
\hline 45111.68 & 45400 & -288 & $7 / 2$ & $135 d^{7}\left({ }^{4} F\right) 6 s 6 p{ }^{4} G+125 d^{7}\left({ }^{4} F\right) 6 s 6 p{ }^{4} G+115 d^{8}\left({ }^{3} F\right) 6 p{ }^{4} G$ \\
\hline 45185.95 & 45210 & -24 & $5 / 2$ & $155 d^{8}\left({ }^{3} F\right) 6 p{ }^{4} D+105 d^{7}\left({ }^{4} F\right) 6 s 6 p{ }^{4} D+65 d^{8}\left({ }^{3} F\right) 6 p{ }^{2} F$ \\
\hline 45259.14 & 45186 & 73 & $3 / 2$ & $135 \mathrm{~d}^{7}\left({ }^{4} \mathrm{P}\right) 6 \mathrm{~s} 6 \mathrm{p}{ }^{6} \mathrm{P}+125 \mathrm{~d}^{8}\left({ }^{3} \mathrm{~F}\right) 6 \mathrm{p}{ }^{4} \mathrm{~F}+85 \mathrm{~d}^{7}\left({ }^{4} \mathrm{~F}\right) 6 \mathrm{~s} 6 \mathrm{p}{ }^{4} \mathrm{~F}$ \\
\hline 45415.26 & 45392 & 23 & $1 / 2$ & $135 \mathrm{~d}^{7}\left({ }^{4} \mathrm{P}\right) 6 \mathrm{~s} 6 \mathrm{p}{ }^{6} \mathrm{D}+125 \mathrm{~d}^{8}\left({ }^{3} \mathrm{~F}\right) 6 \mathrm{p}{ }^{4} \mathrm{D}$ \\
\hline 45503.15 & 45760 & -256 & $1 / 2$ & $115 \mathrm{~d}^{7}\left({ }^{4} \mathrm{P}\right) 6 \mathrm{~s} 6 \mathrm{p}{ }^{6} \mathrm{D}+85 \mathrm{~d}^{7}\left({ }^{2} \mathrm{P}\right) 6 \mathrm{~s} 6 \mathrm{p}{ }^{2} \mathrm{~S}+65 \mathrm{~d}^{8}\left({ }^{3} \mathrm{P}\right) 6 \mathrm{p}{ }^{4} \mathrm{P}$ \\
\hline 45570.89 & 45638 & -67 & $5 / 2$ & $115 d^{7}\left({ }^{4} F\right) 6 s 6 p{ }^{4} G+95 d^{7}\left({ }^{4} F\right) 6 s 6 p{ }^{2} D$ \\
\hline 45895.85 & 46030 & -134 & $7 / 2$ & $285 d^{8}\left({ }^{3} F\right) 6 p{ }^{2} F+125 d^{7}\left({ }^{4} F\right) 6 s 6 p{ }^{4} F+105 d^{7}\left({ }^{4} F\right) 6 s 6 p{ }^{2} F$ \\
\hline 45957.33 & 45502 & 455 & $11 / 2$ & $345 \mathrm{~d}^{7}\left({ }^{2} \mathrm{G}\right) 6 \mathrm{~s} 6 \mathrm{p}{ }^{4} \mathrm{H}+205 \mathrm{~d}^{7}\left({ }^{2} \mathrm{H}\right) 6 \mathrm{~s} 6 \mathrm{p}{ }^{4} \mathrm{I}+125 \mathrm{~d}^{7}\left({ }^{2} \mathrm{G}\right) 6 \mathrm{~s} 6 \mathrm{p}{ }^{4} \mathrm{G}$ \\
\hline 46093.84 & 46259 & -165 & $5 / 2$ & $135 d^{7}\left({ }^{4} F\right) 6 s 6 p{ }^{4} G+85 d^{7}\left({ }^{4} F\right) 6 s 6 p{ }^{4} G+75 d^{7}\left({ }^{4} F\right) 6 s 6 p{ }^{2} D$ \\
\hline 46220.32 & 46011 & 209 & $9 / 2$ & $195 d^{7}\left({ }^{4} F\right) 6 s 6 p{ }^{4} F+115 d^{7}\left({ }^{2} G\right) 6 s 6 p{ }^{4} H+115 d^{6}\left({ }^{5} D\right) 6 s^{2} 6 p{ }^{4} F$ \\
\hline
\end{tabular}


Table 2

(Continued)

\begin{tabular}{|c|c|c|c|c|}
\hline $\begin{array}{l}E_{\exp ^{\mathrm{a}}} \\
\left(\mathrm{cm}^{-1}\right)\end{array}$ & $\begin{array}{l}E_{\mathrm{calc}}^{\mathrm{b}} \\
\left(\mathrm{cm}^{-1}\right)\end{array}$ & $\begin{array}{c}\Delta E \\
\left(\mathrm{~cm}^{-1}\right)\end{array}$ & $J$ & $\begin{array}{l}\text { LS Composition }{ }^{\mathrm{b}, \mathrm{c}} \\
(\%)\end{array}$ \\
\hline 46371.64 & 46371 & 1 & $9 / 2$ & $335 d^{7}\left({ }^{4} P\right) 6 s 6 p{ }^{6} D+125 d^{7}\left({ }^{4} F\right) 6 s 6 p{ }^{4} G+105 d^{7}\left({ }^{2} G\right) 6 s 6 p{ }^{4} F$ \\
\hline 46471.84 & 46433 & 39 & $3 / 2$ & $145 d^{7}\left({ }^{2} P\right) 6 s 6 p{ }^{2} D+125 d^{7}\left({ }^{4} P\right) 6 s 6 p{ }^{4} D+95 d^{7}\left({ }^{4} P\right) 6 s 6 p{ }^{4} S$ \\
\hline 46618.13 & 46696 & -78 & $3 / 2$ & $195 d^{8}\left({ }^{3} F\right) 6 p{ }^{4} D+95 d^{7}\left({ }^{4} F\right) 6 s 6 p^{4} F+75 d^{7}\left({ }^{4} F\right) 6 s 6 p{ }^{4} D$ \\
\hline 46979.02 & 46984 & -5 & $7 / 2$ & $115 d^{6}\left({ }^{5} D\right) 6 s^{2} 6 p{ }^{4} D+95 d^{8}\left({ }^{3} F\right) 6 p{ }^{2} G+95 d^{8}\left({ }^{3} F\right) 6 p{ }^{4} F$ \\
\hline 47011.09 & 47005 & 6 & $5 / 2$ & $155 \mathrm{~d}^{8}\left({ }^{3} \mathrm{~F}\right) 6 \mathrm{p}{ }^{4} \mathrm{G}+115 \mathrm{~d}^{7}\left({ }^{2} \mathrm{~F}\right) 6 \mathrm{~s} 6 \mathrm{p}{ }^{4} \mathrm{G}+55 \mathrm{~d}^{7}\left({ }^{2} \mathrm{H}\right) 6 \mathrm{~s} 6 \mathrm{p}{ }^{4} \mathrm{G}$ \\
\hline 47165.12 & 47349 & -184 & $7 / 2$ & $135 d^{6}\left({ }^{5} \mathrm{D}\right) 6 \mathrm{~s}^{2} 6 \mathrm{p}{ }^{6} \mathrm{~F}+105 \mathrm{~d}^{7}\left({ }^{4} \mathrm{P}\right) 6 \mathrm{~s} 6 \mathrm{p}{ }^{4} \mathrm{D}+65 \mathrm{~d}^{8}\left({ }^{1} \mathrm{D}\right) 6 \mathrm{p}{ }^{2} \mathrm{~F}$ \\
\hline 47203.81 & 47141 & 63 & $1 / 2$ & $235 d^{6}\left({ }^{5} D\right) 6 s^{2} 6 p{ }^{6} D+185 d^{7}\left({ }^{4} P\right) 6 s 6 p{ }^{6} D+165 d^{7}\left({ }^{2} P\right) 6 s 6 p{ }^{2} S$ \\
\hline 47205.57 & 46930 & 276 & $9 / 2$ & $215 d^{7}\left({ }^{4} \mathrm{~F}\right) 6 \mathrm{~s} 6 \mathrm{p}^{2} \mathrm{G}+85 \mathrm{~d}^{7}\left({ }^{2} \mathrm{H}\right) 6 \mathrm{~s} 6 \mathrm{p}{ }^{4} \mathrm{I}+85 \mathrm{~d}^{7}\left({ }^{2} \mathrm{G}\right) 6 \mathrm{~s} 6 \mathrm{p}{ }^{4} \mathrm{~F}$ \\
\hline 47537.29 & 47608 & -71 & $5 / 2$ & $145 d^{6}\left({ }^{5} D\right) 6 s^{2} 6 p{ }^{6} F+95 d^{6}\left({ }^{5} D\right) 6 s^{2} 6 p{ }^{6} D$ \\
\hline 47548.69 & 47685 & -136 & $7 / 2$ & $95 d^{7}\left({ }^{4} F\right) 6 s 6 p{ }^{4} F+75 d^{7}\left({ }^{4} P\right) 6 s 6 p{ }^{6} P+55 d^{7}\left({ }^{4} F\right) 6 s 6 p{ }^{4} D$ \\
\hline 47824.93 & 47744 & 81 & $3 / 2$ & $95 d^{7}\left({ }^{4} P\right) 6 s 6 p{ }^{4} S+95 d^{6}\left({ }^{5} D\right) 6 s^{2} 6 p{ }^{6} D$ \\
\hline 47858.47 & 47889 & -31 & $11 / 2$ & $475 d^{6}\left({ }^{5} D\right) 6 s^{2} 6 p{ }^{6} F+175 d^{7}\left({ }^{4} F\right) 6 s 6 p{ }^{4} G+95 d^{6}\left({ }^{3} F\right) 6 s^{2} 6 p{ }^{4} G$ \\
\hline 48206.57 & 48468 & -261 & $5 / 2$ & $95 d^{7}\left({ }^{4} F\right) 6 s 6 p{ }^{4} F+75 d^{8}\left({ }^{3} P\right) 6 p{ }^{4} P+65 d^{7}\left({ }^{4} P\right) 6 s 6 p{ }^{6} D$ \\
\hline 48299.24 & 48147 & 152 & $9 / 2$ & $235 d^{8}\left({ }^{3} F\right) 6 p^{4} G+95 d^{7}\left({ }^{2} F\right) 6 s 6 p{ }^{4} G+85 d^{8}\left({ }^{3} F\right) 6 p^{2} G$ \\
\hline 48440.83 & 48440 & 1 & $3 / 2$ & $145 d^{6}\left({ }^{5} D\right) 6 s^{2} 6 p{ }^{6} F+95 d^{6}\left({ }^{5} D\right) 6 s^{2} 6 p{ }^{6} D+85 d^{7}\left({ }^{4} F\right) 6 s 6 p{ }^{2} D$ \\
\hline 48448.65 & 48598 & -149 & $7 / 2$ & $395 d^{7}\left({ }^{2} G\right) 6 s 6 p{ }^{4} H+65 d^{7}\left({ }^{4} F\right) 6 s 6 p{ }^{2} F+55 d^{8}\left({ }^{3} F\right) 6 p{ }^{4} G$ \\
\hline 48629.22 & 48690 & -61 & $7 / 2$ & $175 d^{7}\left({ }^{4} F\right) 6 s 6 p{ }^{4} D+135 d^{6}\left({ }^{5} D\right) 6 s^{2} 6 p{ }^{6} F+75 d^{6}\left({ }^{5} D\right) 6 s^{2} 6 p{ }^{4} D$ \\
\hline 48801.91 & 49027 & -225 & $3 / 2$ & $95 d^{7}\left({ }^{4} F\right) 6 s 6 p{ }^{4} F+85 d^{8}\left({ }^{3} F\right) 6 p{ }^{4} F+75 d^{7}\left({ }^{4} F\right) 6 s 6 p{ }^{4} D$ \\
\hline 49146.44 & 49234 & -88 & $5 / 2$ & $115 d^{7}\left({ }^{4} P\right) 6 s 6 p{ }^{6} P+85 d^{7}\left({ }^{4} F\right) 6 s 6 p{ }^{4} D+95 d^{7}\left({ }^{4} F\right) 6 s 6 p{ }^{6} D$ \\
\hline 49158.61 & 49570 & -411 & $9 / 2$ & $215 d^{6}\left({ }^{5} D\right) 6 s^{2} 6 p{ }^{6} D+125 d^{6}\left({ }^{5} D\right) 6 s^{2} 6 p{ }^{4} F+115 d^{6}\left({ }^{5} D\right) 6 s^{2} 6 p{ }^{6} F$ \\
\hline 49342.51 & 49466 & -123 & $3 / 2$ & $195 d^{7}\left({ }^{4} \mathrm{P}\right) 6 \mathrm{~s} 6 \mathrm{p}{ }^{4} \mathrm{~S}+125 \mathrm{~d}^{7}\left({ }^{4} \mathrm{P}\right) 6 \mathrm{~s} 6 \mathrm{p}{ }^{6} \mathrm{P}+65 \mathrm{~d}^{8}\left({ }^{3} \mathrm{P}\right) 6 \mathrm{p}{ }^{4} \mathrm{P}$ \\
\hline 49446.25 & 49353 & 93 & $1 / 2$ & $155 d^{7}\left({ }^{4} \mathrm{P}\right) 6 \mathrm{~s} 6 \mathrm{p}{ }^{4} \mathrm{D}+135 \mathrm{~d}^{7}\left({ }^{4} \mathrm{P}\right) 6 \mathrm{~s} 6 \mathrm{p}{ }^{4} \mathrm{P}+105 \mathrm{~d}^{7}\left({ }^{4} \mathrm{~F}\right) 6 \mathrm{~s} 6 \mathrm{p}{ }^{4} \mathrm{D}$ \\
\hline 49719.17 & 48977 & 742 & $11 / 2$ & $335 d^{6}\left({ }^{5} D\right) 6 s^{2} 6 p{ }^{6} F+185 d^{7}\left({ }^{2} G\right) 6 s 6 p{ }^{4} G+155 d^{7}\left({ }^{4} F\right) 6 s 6 p{ }^{4} G$ \\
\hline 49779.37 & 49895 & -116 & $5 / 2$ & $125 d^{7}\left({ }^{4} P\right) 6 s 6 p{ }^{4} P+75 d^{8}\left({ }^{3} P\right) 6 p{ }^{4} D+65 d^{7}\left({ }^{4} F\right) 6 s 6 p{ }^{4} D$ \\
\hline 49823.54 & 49765 & 59 & $7 / 2$ & $115 d^{8}\left({ }^{3} F\right) 6 p^{2} G+115 d^{6}\left({ }^{5} D\right) 6 s^{2} 6 p{ }^{6} F+115 d^{7}\left({ }^{2} G\right) 6 s 6 p{ }^{4} F$ \\
\hline
\end{tabular}

Notes.

${ }^{\mathrm{a}}$ From Kramida et al. (2018).

b This work.

${ }^{c}$ Only the first three eigenvector components are given, provided they are greater than or equal to $5 \%$.

above $50,000 \mathrm{~cm}^{-1}$ were not included in the fit because many of them were found to be strongly mixed with unknown levels, which made very doubtful the correspondence between the calculated energies and the available experimental values. For this reason, our HFR + CPOL calculations of the atomic structure and radiative parameters in Ir I were limited to the energy levels situated below $50,000 \mathrm{~cm}^{-1}$. The energy levels computed in our work are compared to the available experimental values in Tables 1 and 2 for even and odd parities, respectively. As seen from these tables, a good agreement is obtained between both sets of results, the average deviations being found to be equal to $59 \mathrm{~cm}^{-1}$ (even parity) and $112 \mathrm{~cm}^{-1}$ (odd parity). We can also note that the levels considered in the present study are extremely mixed, in particular for the odd parity for which very strong intermediate coupling and configuration interaction among $5 d^{8} 6 p, 5 d^{7} 6 s 6 p$, and $5 d^{6} 6 s^{2} 6 p$ are observed, as shown in Table 2. This HFR+CPOL approach was then used to compute the radiative decay rates for the transitions depopulating the Ir I odd energy levels located below $50,000 \mathrm{~cm}^{-1}$. All the line strengths were calculated in the length form.

\section{Results and Discussion}

The lifetimes measured in the present work for 62 odd-parity levels of $\mathrm{Ir} I$ in the region of $32513.43-58625.10 \mathrm{~cm}^{-1}$ are listed in Table 3. Among them, three levels were also studied in the literature through experiments and calculations, thus their previous results are presented for comparison. For the three levels previously reported, it is seen that our results are in rather good agreement with the values measured by Gough et al. (1983) and Xu et al. (2007) using the TR-LIF technique. For the $33874.43 \mathrm{~cm}^{-1}$ level, the result reported by Ramanujam \& Andersen (1978) is $34 \pm 3 \mathrm{ns,} \mathrm{which} \mathrm{shows} \mathrm{a} \mathrm{slightly} \mathrm{larger}$ difference with our value of $28.4 \pm 0.6 \mathrm{~ns}$ and the other two results in the literature. Considering that the former was measured by the beam-sputtering excitation method, which might involve a cascade population for the studied level and hence prolonging its decay time if cascade correction is not performed well, it is reasonable to believe that our result is more reliable.

In Table 3, except for the three energy levels with previous results, to our best knowledge the other 59 levels are experimentally measured for the first time. The measured lifetime values are in the range from 3.2 to $345 \mathrm{~ns}$, and their uncertainties are smaller than $10 \%$ (except $10.5 \%$ for the level at $43529.31 \mathrm{~cm}^{-1}$ ) and more than half of them are within $5 \%$.

Our HFR+CPOL theoretical lifetimes are also given in Table 3 . The general agreement between theory and experiment can be judged as rather satisfactory (within a factor of 2), if we discount the two $J=3 / 2$ levels at 44785.44 and $48440.83 \mathrm{~cm}^{-1}$ for which the calculated lifetimes are respectively a factor of about 4 and 7 longer than the experimental 
Table 3

Measured and Calculated Lifetimes for Ir I Levels and Comparison with Previous Results

\begin{tabular}{|c|c|c|c|c|c|c|c|c|c|}
\hline \multicolumn{2}{|c|}{ Upper Level $^{\mathrm{a}}$} & \multicolumn{2}{|c|}{ Lower Level ${ }^{\mathrm{a}}$} & \multirow{3}{*}{$\begin{array}{l}\lambda_{\text {Exc. }} \\
(\mathrm{nm})\end{array}$} & \multirow{3}{*}{$\begin{array}{l}\lambda_{\text {Obs. }} \\
(\mathrm{nm})\end{array}$} & \multicolumn{4}{|c|}{ Lifetime (ns) } \\
\hline \multirow{2}{*}{ Assignment } & \multirow{2}{*}{$E\left(\mathrm{~cm}^{-1}\right)$} & \multirow{2}{*}{ Assignment } & \multirow{2}{*}{$E\left(\mathrm{~cm}^{-1}\right)$} & & & \multicolumn{2}{|c|}{ Experiment } & \multicolumn{2}{|c|}{ Calculation } \\
\hline & & & & & & This work & $\overline{\text { Previous }}$ & This work & $\overline{\text { Previous }}$ \\
\hline$\overline{5 d^{7} 6 s\left({ }^{5} F\right) 6 p ~ z ~}{ }^{6} F^{\circ} 9 / 2$ & 32513.43 & $5 d^{7} 6 s^{2}$ a ${ }^{4} F_{9 / 2}$ & 0 & 307.565 & 381.832 & $345(11)$ & $335(10)^{\mathrm{b}}$ & 430 & $\begin{array}{l}303^{\mathrm{c}} \\
430^{\mathrm{c}}\end{array}$ \\
\hline $5 d^{7} 6 s\left({ }^{5} F\right) 6 p z^{6} F^{\circ}{ }_{7 / 2}$ & 33874.43 & $5 d^{8}\left({ }^{3} F\right) 6 s ~ b ~{ }^{4} F_{9 / 2}$ & 2834.98 & 322.171 & 362.97 & $28.4(6)$ & $\begin{array}{c}27.5(10)^{\mathrm{b}} \\
29.6(20)^{\mathrm{c}} \\
34(3)^{\mathrm{d}}\end{array}$ & 21.2 & $\begin{array}{l}15.9^{\mathrm{c}} \\
21.2^{\mathrm{c}}\end{array}$ \\
\hline $5 d^{7} 6 s\left({ }^{5} F\right) 6 p z^{6} G^{\circ}{ }_{5 / 2}$ & 35540.34 & $5 d^{7} 6 s^{2}$ a ${ }^{4} F_{3 / 2}$ & 4078.94 & 317.85 & 351.695 & $281(15)$ & & 205 & \\
\hline $5 d^{7} 6 s\left({ }^{5} \mathrm{~F}\right) 6 \mathrm{p} \mathrm{z}{ }^{4} \mathrm{D}^{\circ}{ }_{5 / 2}$ & 37446.13 & $5 d^{7} 6 s^{2}$ a ${ }^{4} F_{5 / 2}$ & 5784.62 & 315.841 & 362.732 & $194(15)$ & $180(10)^{\mathrm{b}}$ & 128 & $\begin{array}{c}94.0^{\mathrm{c}} \\
128^{\mathrm{c}}\end{array}$ \\
\hline $4052^{\circ}{ }_{3 / 2}$ & 40524.73 & $5 \mathrm{~d}^{7} 6 \mathrm{~s}^{2}$ a ${ }^{4} \mathrm{~F}_{5 / 2}$ & 5784.62 & 287.852 & 356.9 & $20.4(14)$ & & 43.1 & \\
\hline $4152^{\circ}{ }_{5 / 2}$ & 41522.22 & $5 d^{8}\left({ }^{3} F\right) 6 s$ b ${ }^{4} F_{5 / 2}$ & 9877.54 & 316.009 & 267.071 & $16.8(5)$ & & 19.6 & \\
\hline $4226^{\circ} 5 / 2$ & 42267.86 & $5 d^{8}\left({ }^{3} \mathrm{~F}\right) 6 \mathrm{~s} \mathrm{~b}{ }^{4} \mathrm{~F}_{5 / 2}$ & 9877.54 & 308.734 & 261.856 & $23.3(13)$ & & 28.7 & \\
\hline $4227^{\circ} 9 / 2$ & 42279.28 & $5 d^{8}\left({ }^{3} F\right) 6 s$ b ${ }^{4} F_{7 / 2}$ & 7106.61 & 284.312 & 253.522 & $74.0(27)$ & & 57.7 & \\
\hline $4317^{\circ} 7 / 2$ & 43176.15 & $5 d^{7} 6 s^{2}$ a ${ }^{4} F_{9 / 2}$ & 0 & 231.609 & 300.313 & 19.7(9) & & 26.7 & \\
\hline $4359^{\circ}{ }_{7 / 2}$ & 43529.21 & $5 d^{7} 6 s^{2}$ a ${ }^{4} F_{9 / 2}$ & 0 & 229.399 & 264.497 & $53.2(56)$ & & 50.4 & \\
\hline $4459^{\circ}{ }_{5 / 2}$ & 44596.77 & $5 d^{8}\left({ }^{3} F\right) 6 s b{ }^{4} F_{5 / 2}$ & 9877.54 & 288.025 & 356.743 & $17.5(14)$ & & 20.5 & \\
\hline $4464^{\circ} 7 / 2$ & 44642.67 & $5 d^{8}\left({ }^{3} \mathrm{~F}\right) 6 s$ a ${ }^{2} \mathrm{~F}_{5 / 2}$ & 12218.47 & 308.412 & 224.001 & $7.7(5)$ & & 15.6 & \\
\hline $4465^{\circ} 9 / 2$ & 44652.43 & $5 d^{7} 6 s^{2}$ a ${ }^{4} F_{9 / 2}$ & 0 & 223.952 & 266.341 & $12.2(8)$ & & 27.5 & \\
\hline $4478^{\circ}{ }_{3 / 2}$ & 44785.44 & $5 d^{8}\left({ }^{3} \mathrm{~F}\right) 6 \mathrm{~s} \mathrm{~b}{ }^{4} \mathrm{~F}_{5 / 2}$ & 9877.54 & 286.468 & 354.357 & $7.1(4)$ & & 28.0 & \\
\hline $4511^{\circ} 7 / 2$ & 45111.68 & $5 d^{8}\left({ }^{3} \mathrm{~F}\right) 6 \mathrm{~s} \mathrm{~b}{ }^{4} \mathrm{~F}_{5 / 2}$ & 9877.54 & 283.816 & 304.014 & $17.9(8)$ & & 29.0 & \\
\hline $4518^{\circ}{ }_{5 / 2}$ & 45185.95 & $5 d^{8}\left({ }^{3} \mathrm{~F}\right) 6 \mathrm{~s}$ a ${ }^{2} \mathrm{~F}_{7 / 2}$ & 13087.90 & 311.545 & 243.268 & $10.4(3)$ & & 10.0 & \\
\hline $4525^{\circ}{ }_{3 / 2}$ & 45259.14 & $5 d^{8}\left({ }^{3} F\right) 6 s$ b ${ }^{4} F_{5 / 2}$ & 9877.54 & 282.633 & 305.311 & $16.8(5)$ & & 20.2 & \\
\hline $4557^{\circ} 5 / 2$ & 45570.89 & $5 d^{8}\left({ }^{3} F\right) 6 s b{ }^{4} F_{5 / 2}$ & 9877.54 & 280.164 & 306.568 & 13.1(4) & & 11.7 & \\
\hline $4589^{\circ} 7 / 2$ & 45895.85 & $5 d^{7} 6 s^{2}$ a ${ }^{4} F_{9 / 2}$ & 0 & 217.885 & 312.93 & $7.6(2)$ & & 9.8 & \\
\hline $4622^{\circ} 9 / 2$ & 46220.32 & $5 d^{7} 6 s^{2}$ a ${ }^{4} F_{9 / 2}$ & 0 & 216.355 & 250.649 & $11.4(6)$ & & 26.1 & \\
\hline $4637_{9 / 2}^{\circ}$ & 46371.64 & $5 d^{7} 6 s^{2}$ a ${ }^{4} F_{9 / 2}$ & 0 & 215.649 & 254.68 & $22.6(8)$ & & 21.1 & \\
\hline $4716^{\circ}{ }_{7 / 2}$ & 47165.12 & $5 d^{8}\left({ }^{3} F\right) 6 s$ b ${ }^{4} F_{9 / 2}$ & 2834.98 & 225.58 & 249.635 & $14.3(8)$ & & 16.8 & \\
\hline $4720^{\circ} 9 / 2$ & 47205.57 & $5 d^{7} 6 s^{2}$ a ${ }^{4} F_{9 / 2}$ & 0 & 211.839 & 249.383 & $31.7(11)$ & & 24.8 & \\
\hline $4754^{\circ}{ }_{7 / 2}$ & 47548.69 & $5 d^{7} 6 s^{2}$ a ${ }^{4} F_{9 / 2}$ & 0 & 210.311 & 247.267 & $21.7(7)$ & & 29.8 & \\
\hline $4785^{\circ} 11 / 2$ & 47858.47 & $5 d^{7} 6 s^{2}$ a ${ }^{4} F_{9 / 2}$ & 0 & 208.949 & 410.634 & $3.7(3)$ & & 6.5 & \\
\hline $4820^{\circ}{ }_{5 / 2}$ & 48206.57 & $5 d^{7} 6 s^{2} b{ }^{4} P_{5 / 2}$ & 16103.32 & 311.495 & 260.899 & $8.3(7)$ & & 13.8 & \\
\hline $4844_{3 / 2}^{\circ}$ & 48440.83 & $5 d^{7} 6 s^{2}$ a ${ }^{4} F_{3 / 2}$ & 4078.94 & 225.419 & 264.116 & $6.2(2)$ & & 40.7 & \\
\hline $4844^{\circ}{ }_{7 / 2}^{3 / 2}$ & 48448.65 & $5 d^{7} 6 s^{2}$ a ${ }^{4} F_{9 / 2}$ & 0 & 206.404 & 241.855 & $8.5(3)$ & & 15.3 & \\
\hline $4862^{\circ}{ }_{7 / 2}$ & 48629.22 & $5 d^{7} 6 s^{2}$ a ${ }^{4} F_{9 / 2}$ & 0 & 205.638 & 218.368 & $6.3(3)$ & & 6.6 & \\
\hline $4977^{\circ} 5 / 2$ & 49779.37 & $5 d^{7} 6 s^{2}$ a ${ }^{4} F_{3 / 2}$ & 4078.94 & 218.816 & 255.098 & $11.0(6)$ & & 23.5 & \\
\hline $4982^{\circ} 7 / 2$ & 49823.54 & $5 d^{8}\left({ }^{3} F\right) 6 s b{ }^{4} F_{9 / 2}$ & 2834.98 & 212.818 & 250.338 & $16.2(5)$ & & 12.4 & \\
\hline $5016^{\circ}{ }_{5 / 2}$ & 50169.88 & $5 d^{7} 6 s^{2}$ a ${ }^{4} F_{3 / 2}$ & 4078.94 & 216.962 & 252.581 & $10.1(4)$ & & & \\
\hline $5056_{3 / 2}^{\circ}$ & 50564.12 & $5 d^{7} 6 s^{2}$ a ${ }^{4} F_{3 / 2}$ & 4078.94 & 215.122 & 245.781 & $9.8(3)$ & & & \\
\hline $5058^{\circ}{ }_{7 / 2}$ & 50580.39 & $5 d^{8}\left({ }^{3} F\right) 6 s b{ }^{4} F_{9 / 2}$ & 2834.98 & 209.444 & 230.024 & $8.5(6)$ & & & \\
\hline $5116^{\circ}{ }_{3 / 2}$ & 51166.54 & $5 d^{7} 6 s^{2}$ a ${ }^{4} F_{3 / 2}$ & 4078.94 & 212.37 & 246.379 & $27.6(4)$ & & & \\
\hline $5142^{\circ} 5 / 2$ & 51427.15 & $5 d^{7} 6 s^{2}$ a ${ }^{4} F_{3 / 2}$ & 4078.94 & 211.201 & 252.55 & $11.9(5)$ & & & \\
\hline $5147_{9 / 2}^{\circ}$ & 51470.74 & $5 d^{8}\left({ }^{3} \mathrm{~F}\right) 6 \mathrm{~s} b{ }^{4} \mathrm{~F}_{9 / 2}$ & 2834.98 & 205.61 & 225.407 & $7.8(4)$ & & & \\
\hline $5181^{\circ}{ }_{5 / 2}$ & 51814.75 & $5 d^{7} 6 s^{2}$ a ${ }^{4} F_{3 / 2}$ & 4078.94 & 209.486 & 238.452 & $15.5(4)$ & & & \\
\hline $5198^{\circ}{ }_{1 / 2}$ & 51983.92 & $5 \mathrm{~d}^{7} 6 \mathrm{~s}^{2}$ a ${ }^{4} \mathrm{~F}_{3 / 2}$ & 4078.94 & 208.747 & 241.515 & $13.6(5)$ & & & \\
\hline $5205^{\circ}{ }_{5 / 2}$ & 52051.75 & $5 d^{7} 6 s^{2}$ a ${ }^{4} F_{3 / 2}$ & 4078.94 & 208.451 & 237.112 & $13.5(4)$ & & & \\
\hline $5213^{\circ}{ }_{7 / 2}$ & 52134.11 & $5 \mathrm{~d}^{7} 6 \mathrm{~s}^{2}$ a ${ }^{4} \mathrm{~F}_{5 / 2}$ & 5784.62 & 215.752 & 250.528 & $10.2(2)$ & & & \\
\hline $5222^{\circ} 7 / 2$ & 52224.37 & $5 d^{7} 6 s^{2}$ a ${ }^{4} F_{5 / 2}$ & 5784.62 & 215.333 & 249.963 & $7.1(3)$ & & & \\
\hline $5230^{\circ}{ }_{3 / 2}$ & 52303.65 & $5 d^{7} 6 s^{2}$ a ${ }^{4} F_{3 / 2}$ & 4078.94 & 207.363 & 239.665 & $8.2(3)$ & & & \\
\hline $5232^{\circ}{ }_{5 / 2}$ & 52327.33 & $5 d^{7} 6 s^{2}$ a ${ }^{4} F_{3 / 2}$ & 4078.94 & 207.261 & 235.572 & $7.9(5)$ & & & \\
\hline $5238^{\circ} \quad 1 / 2$ & 52388.38 & $5 d^{7} 6 s^{2}$ a ${ }^{4} F_{3 / 2}$ & 4078.94 & 206.999 & 246.565 & $6.8(4)$ & & & \\
\hline $5260^{\circ}{ }_{5 / 2}$ & 52605.16 & $5 d^{7} 6 s^{2}$ a ${ }^{4} F_{3 / 2}$ & 4078.94 & 206.074 & 234.041 & $22.4(9)$ & & & \\
\hline $5280^{\circ}{ }_{3 / 2}$ & 52806.57 & $5 d^{7} 6 s^{2}$ a ${ }^{4} F_{3 / 2}$ & 4078.94 & 205.222 & 236.81 & $11.7(9)$ & & & \\
\hline $5355^{\circ} 5 / 2$ & 53552.93 & $5 d^{7} 6 s^{2}$ a ${ }^{4} F_{5 / 2}$ & 5784.62 & 209.344 & 241.929 & $5.9(4)$ & & & \\
\hline $5364^{\circ} 9 / 2$ & 53642.06 & $5 d^{7} 6 s^{2}$ a ${ }^{4} F_{7 / 2}$ & 6323.91 & 211.335 & 246.584 & $5.0(3)$ & & & \\
\hline $5368^{\circ} 7 / 2$ & 53686.99 & $5 d^{7} 6 s^{2}$ a ${ }^{4} F_{7 / 2}$ & 6323.91 & 211.135 & 241.147 & $6.4(3)$ & & & \\
\hline $5411_{5 / 2}^{\circ}$ & 54119.23 & $5 \mathrm{~d}^{7} 6 \mathrm{~s}^{2}$ a ${ }^{4} \mathrm{~F}_{5 / 2}$ & 5784.62 & 206.891 & 229.671 & $9.0(5)$ & & & \\
\hline $5414^{\circ} 7 / 2$ & 54140.83 & $5 d^{8}\left({ }^{3} \mathrm{~F}\right) 6 \mathrm{~s} \mathrm{~b}{ }^{4} \mathrm{~F}_{7 / 2}$ & 7106.61 & 212.611 & 248.75 & $5.7(3)$ & & & \\
\hline $5426_{9 / 2}^{\circ}$ & 54263.79 & $5 d^{7} 6 s^{2}$ a ${ }^{4} F_{7 / 2}$ & 6323.91 & 208.595 & 242.861 & $20.3(7)$ & & & \\
\hline $5456^{\circ} 7 / 2$ & 54566.06 & $5 d^{7} 6 s^{2}$ a ${ }^{4} F_{7 / 2}$ & 6323.91 & 207.288 & 236.141 & $6.4(4)$ & & & \\
\hline $5463^{\circ} 5 / 2$ & 54639.31 & $5 d^{7} 6 s^{2}$ a ${ }^{4} F_{7 / 2}$ & 6323.91 & 206.973 & 233.6 & $5.8(4)$ & & & \\
\hline $5466^{\circ} 9 / 2$ & 54667.54 & $5 d^{7} 6 s^{2}$ a ${ }^{4} F_{7 / 2}$ & 6323.91 & 206.852 & 320.907 & $21.0(17)$ & & & \\
\hline $5471^{\circ} 7 / 2$ & 54711.10 & $5 d^{8}\left({ }^{3} F\right) 6 s b^{4} F_{7 / 2}$ & 7106.61 & 210.064 & 259.015 & $7.7(5)$ & & & \\
\hline $5489^{\circ}{ }_{7 / 2}$ & 54894.82 & $5 d^{7} 6 s^{2}$ a ${ }^{4} F_{5 / 2}$ & 5784.62 & 203.624 & 257.788 & $11.8(6)$ & & & \\
\hline
\end{tabular}


Table 3

(Continued)

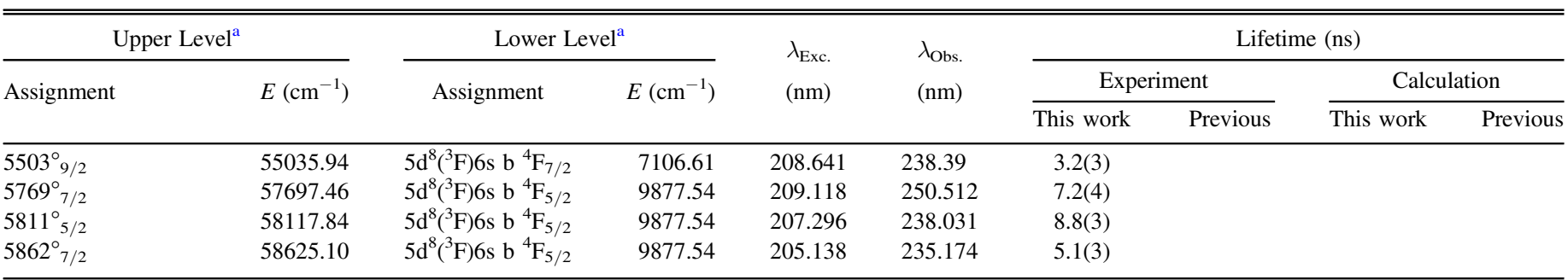

Notes. The number in parentheses is the uncertainty in the last one or two digits of the reported result.

${ }^{\text {a }}$ Kramida et al. (2018).

${ }^{\mathrm{b}}$ Gough et al. (1983).

${ }^{\mathrm{c}} \mathrm{Xu}$ et al. (2007).

${ }^{\mathrm{d}}$ Ramanujam \& Anderson (1978).

values. It is worth noting that, according to our calculations, most of the highly excited levels considered in the present work were found to be extremely mixed with, on average, a main LS component as low as $20 \%$ for all the levels listed in Table 3, and this main component not exceeding even $15 \%$ for the levels located between 40,000 and $50,000 \mathrm{~cm}^{-1}$.

Using the theoretical BFs and the experimental lifetimes, we deduced the transition probabilities and oscillator strengths for all the lines depopulating the odd-parity levels below $50,000 \mathrm{~cm}^{-1}$. The results obtained are reported in Table 4 . These correspond to 134 Ir I spectral lines appearing in the wavelength range from 205 to $418 \mathrm{~nm}$. Note that concerning the BFs, only the values larger than 0.05 have been retained in the table. To our knowledge, transition probabilities were experimentally measured by Gough et al. (1983) for only two transitions among those considered in the present work. These are found to be in good agreement (within 20\%) with our values.

The estimated uncertainties of the transition probabilities and oscillator strengths obtained in our work are also reported in Table 4, using the letter coding as the one usually employed in the NIST database (Kramida et al. 2018). They were evaluated as follows. First, an uncertainty was assigned to all our calculated HFR +CPOL BF values by comparing the latter to those deduced from experimental measurements by Gough et al. (1983) and Ivarsson et al. (2003) for some transitions depopulating Ir I levels up to $40,710 \mathrm{~cm}^{-1}$. Such a comparison is reported in Table 7 of $\mathrm{Xu}$ et al. (2007) and illustrated in Figure 3 of the present paper where the relative differences
$\left(\mathrm{BF}_{\text {calc }}-\mathrm{BF}_{\text {exp }}\right) / \mathrm{BF}_{\text {calc }}$ are reported against $\mathrm{BF}_{\text {calc. When }}$ looking at this figure, one can clearly note a rather regular pattern of increasingly deviating weak branches, the average uncertainties on calculated BF-values being found to be about $10 \%$, for $0.8<\mathrm{BF}_{\text {calc }}<1.0,20 \%$ for $0.6<\mathrm{BF}_{\text {calc }}<0.8$, $30 \%$ for $0.4<\mathrm{BF}_{\text {calc }}<0.6,40 \%$ for $0.2<\mathrm{BF}_{\text {calc }}<0.4$ and $100 \%$ for $0.0<\mathrm{BF}_{\text {calc }}<0.2$. These uncertainties were then combined in quadrature with the experimental lifetime uncertainties derived from our measurements to yield the uncertainties of $g A$ - and $g f$-values. As a final result, out of the 134 transitions listed in Table 4, 46 have an estimated decay rate accuracy that is better than $50 \%$, which illustrates the fact that many of the highly excited levels considered in the present work are depopulated by quite a number of weak transitions. Moreover, a few transitions reported in Table 4 with an asterisk were found to be affected by strong cancellation effects in our HFR + CPOL calculations. More precisely, for such transitions, the cancellation factor (CF), as defined by Cowan (1981), was estimated to be smaller than 0.01 , indicating that the corresponding line strength, and thus the BF or transition probability, might be expected to show a larger percentage uncertainty.

This work was supported by the Science and Technology Development Planning Project of Jilin Province (Grant No. 20180101239JC). P.P. and P.Q. are respectively Research Associate and Research Director of the Belgian F.R.S.-FNRS, from which financial support is gratefully acknowledged. 
Table 4

Branching Fractions, Transition Probabilities, and Oscillator Strengths Obtained in the Present Work for Highly Excited Levels of Ir I, and Comparison with Previous Results

\begin{tabular}{|c|c|c|c|c|c|c|c|c|c|c|}
\hline \multicolumn{2}{|c|}{ Upper Level $^{\mathrm{a}}$} & \multicolumn{2}{|c|}{ Lower Level $^{\mathrm{a}}$} & \multirow{3}{*}{$\lambda_{\text {air }}(\mathrm{nm})$} & \multirow{3}{*}{$\mathrm{BF}^{\mathrm{b}}$} & \multicolumn{2}{|c|}{$g A\left(10^{6} \mathrm{~s}^{-1}\right)$} & \multicolumn{3}{|c|}{$\log (g f)$} \\
\hline \multirow{2}{*}{ Assign. } & \multirow{2}{*}{$E\left(\mathrm{~cm}^{-1}\right)$ Lifetime (ns) } & \multirow{2}{*}{ Assign. } & \multirow{2}{*}{$E\left(\mathrm{~cm}^{-1}\right)$} & & & \multirow{2}{*}{ This work ${ }^{\mathrm{b}}$} & \multirow{2}{*}{$\begin{array}{l}\text { Previous } \\
\text { Exp. }\end{array}$} & \multirow{2}{*}{ This work ${ }^{\mathrm{c}}$} & \multicolumn{2}{|c|}{ Previous } \\
\hline & & & & & & & & & Exp. & Calc. \\
\hline $5 \mathrm{~d}^{7} 6 \mathrm{~s}\left({ }^{5} \mathrm{~F}\right) 6 \mathrm{p} \mathrm{z}{ }^{6} \mathrm{~F}_{9 / 2}^{\circ}$ & $\begin{array}{c}32513.43 \\
\tau=345(11)\end{array}$ & $\begin{array}{c}5 d^{7} 6 s^{2} \text { a }{ }^{4} \mathrm{~F}_{9 / 2} \\
5 d^{8}\left({ }^{3} \mathrm{~F}\right) 6 \mathrm{~s} \mathrm{~b}{ }^{4} \mathrm{~F}_{9 / 2} \\
5 \mathrm{~d}^{7} 6 \mathrm{~s}^{2} \text { a }{ }^{4} \mathrm{~F}_{7 / 2} \\
5 \mathrm{~d}^{8}\left({ }^{3} \mathrm{~F}\right) 6 \mathrm{~s} \text { b }{ }^{4} \mathrm{~F}_{7 / 2}\end{array}$ & $\begin{array}{c}0.00 \\
2834.98 \\
6323.91 \\
7106.61\end{array}$ & $\begin{array}{l}307.476 \\
336.848 \\
381.724 \\
393.484\end{array}$ & $\begin{array}{c}0.10^{*} \\
0.21 \\
0.17 \\
0.51\end{array}$ & $\begin{array}{c}2.89(E) \\
6.09(D+) \\
4.93(E) \\
14.7(D+)\end{array}$ & & $\begin{array}{c}-2.38(E) \\
-1.98(D+) \\
-1.97(E) \\
-1.47(D+)\end{array}$ & & $\begin{array}{l}-2.34^{\mathrm{d}} \\
-1.98^{\mathrm{d}} \\
-1.96^{\mathrm{d}} \\
-1.45^{\mathrm{d}}\end{array}$ \\
\hline $5 \mathrm{~d}^{7} 6 \mathrm{~s}\left({ }^{5} \mathrm{~F}\right) 6 \mathrm{p} \mathrm{z}{ }^{6} \mathrm{~F}^{\circ}{ }_{7 / 2}$ & $\begin{array}{c}33874.43 \\
\tau=28.4(6)\end{array}$ & $\begin{array}{c}5 d^{8}\left({ }^{3} \mathrm{~F}\right) 6 \mathrm{~s} \mathrm{~b}{ }^{4} \mathrm{~F}_{9 / 2} \\
5 \mathrm{~d}^{7} 6 \mathrm{~s}^{2} \text { a }{ }^{4} \mathrm{~F}_{7 / 2}\end{array}$ & $\begin{array}{l}2834.98 \\
6323.91\end{array}$ & $\begin{array}{l}322.078 \\
362.867\end{array}$ & $\begin{array}{l}0.80 \\
0.06\end{array}$ & $\begin{array}{l}225(B) \\
16.9(E)\end{array}$ & $\begin{array}{c}192.9^{\mathrm{e}} \\
22.7^{\mathrm{e}}\end{array}$ & $\begin{array}{l}-0.46(B) \\
-1.48(E)\end{array}$ & $\begin{array}{l}-0.523^{\mathrm{d}} \\
-1.35^{\mathrm{d}}\end{array}$ & $\begin{array}{l}-0.47^{\mathrm{d}} \\
-1.51^{\mathrm{d}}\end{array}$ \\
\hline $5 \mathrm{~d}^{7} 6 \mathrm{~s}\left({ }^{5} \mathrm{~F}\right) 6 \mathrm{p} \mathrm{z}{ }^{6} \mathrm{G}_{5 / 2}^{\circ}$ & $\begin{array}{c}35540.34 \\
\tau=281(15)\end{array}$ & $\begin{array}{c}5 d^{7} 6 s^{2} \text { a }{ }^{4} F_{3 / 2} \\
5 d^{8}\left({ }^{3} \mathrm{~F}\right) 6 s \text { b b }{ }^{4} \mathrm{~F}_{7 / 2}\end{array}$ & $\begin{array}{l}4078.94 \\
7106.61\end{array}$ & $\begin{array}{l}317.758 \\
351.594\end{array}$ & $\begin{array}{l}0.60 \\
0.23\end{array}$ & $\begin{array}{l}12.8(D+) \\
4.91(D+)\end{array}$ & & $\begin{array}{l}-1.71(D+) \\
-2.05(D+)\end{array}$ & & \\
\hline $5 \mathrm{~d}^{7} 6 \mathrm{~s}\left({ }^{5} \mathrm{~F}\right) 6 \mathrm{p} \mathrm{z}{ }^{4} \mathrm{D}_{5 / 2}^{\circ}$ & $\begin{array}{c}37446.13 \\
\tau=194(15)\end{array}$ & $\begin{array}{c}5 d^{7} 6 s^{2} \text { a }{ }^{4} F_{3 / 2} \\
5 d^{7} 6 s^{2} \text { a }{ }^{4} F_{5 / 2} \\
5 d^{7} 6 s^{2} \text { a }{ }^{4} \mathrm{~F}_{7 / 2} \\
5 d^{8}\left({ }^{3} \mathrm{~F}\right) 6 s \text { b }{ }^{4} \mathrm{~F}_{5 / 2} \\
5 d^{8}\left({ }^{3} \mathrm{~F}\right) 6 s \text { b }{ }^{4} \mathrm{~F}_{3 / 2}\end{array}$ & $\begin{array}{c}4078.94 \\
5784.62 \\
6323.91 \\
9877.54 \\
11831.09\end{array}$ & $\begin{array}{l}299.609 \\
315.750 \\
321.221 \\
362.629 \\
390.285\end{array}$ & $\begin{array}{l}0.44 \\
0.11 \\
0.11 \\
0.22 \\
0.09\end{array}$ & $\begin{array}{c}13.6(D+) \\
3.40(E) \\
3.40(E) \\
6.80(D+) \\
2.78(E)\end{array}$ & & $\begin{array}{c}-1.74(D+) \\
-2.29(E) \\
-2.28(E) \\
-1.87(D+) \\
-2.20(E)\end{array}$ & & $\begin{array}{l}-1.70^{\mathrm{d}} \\
-2.28^{\mathrm{d}} \\
-2.36^{\mathrm{d}} \\
-1.85^{\mathrm{d}} \\
-2.16^{\mathrm{d}}\end{array}$ \\
\hline $4052^{\circ}{ }_{3 / 2}$ & $\begin{array}{c}40524.73 \\
\tau=20.4(14)\end{array}$ & $\begin{array}{c}5 \mathrm{~d}^{7} 6 \mathrm{~s}^{2} \text { a }{ }^{4} \mathrm{~F}_{5 / 2} \\
5 \mathrm{~d}^{8}\left({ }^{3} \mathrm{~F}\right) 6 \mathrm{~s} \text { b }{ }^{4} \mathrm{~F}_{5 / 2} \\
5 \mathrm{~d}^{8}\left({ }^{3} \mathrm{P}\right) 6 \mathrm{~s} \text { a }{ }^{2} \mathrm{P}_{3 / 2} \\
5 \mathrm{~d}^{8}\left({ }^{3} \mathrm{P}\right) 6 \mathrm{~s} \text { a }{ }^{2} \mathrm{P}_{1 / 2} \\
5 \mathrm{~d}^{8}\left({ }^{3} \mathrm{P}\right) 6 \mathrm{~s} \text { a }{ }^{4} \mathrm{P}_{5 / 2} \\
5 \mathrm{~d}^{8}\left({ }^{3} \mathrm{P}\right) 6 \mathrm{~s} \text { a }{ }^{4} \mathrm{P}_{3 / 2}\end{array}$ & $\begin{array}{c}5784.62 \\
9877.54 \\
10578.68 \\
12505.68 \\
12951.67 \\
16565.35\end{array}$ & $\begin{array}{l}287.768 \\
326.200 \\
333.838 \\
356.798 \\
362.570 \\
417.255\end{array}$ & $\begin{array}{l}0.30 \\
0.15 \\
0.19 \\
0.07 \\
0.10 \\
0.14\end{array}$ & $\begin{array}{l}58.8(D+) \\
29.4(E) \\
37.3(E) \\
13.7(E) \\
19.6(E) \\
27.4(E)\end{array}$ & & $\begin{array}{l}-1.14(D+) \\
-1.33(E) \\
-1.21(E) \\
-1.58(E) \\
-1.41(E) \\
-1.14(E)\end{array}$ & & \\
\hline $4152^{\circ} 5 / 2$ & $\begin{array}{c}41522.22 \\
\tau=16.8(5)\end{array}$ & $\begin{array}{c}5 \mathrm{~d}^{7} 6 \mathrm{~s}^{2} \text { a }{ }^{4} \mathrm{~F}_{3 / 2} \\
5 \mathrm{~d}^{7} 6 \mathrm{~s}^{2} \text { a a }{ }^{4} \mathrm{~F}_{5 / 2} \\
5 \mathrm{~d}^{7} 6 \mathrm{~s}^{2} \text { a }{ }^{4} \mathrm{~F}_{7 / 2} \\
\left.5 \mathrm{~d}^{8}{ }^{3} \mathrm{~F}\right) 6 \mathrm{~s} \text { b }{ }^{4} \mathrm{~F}_{7 / 2} \\
5 \mathrm{~d}^{8}\left({ }^{3} \mathrm{~F}\right) 6 \mathrm{~s} \text { b }{ }^{4} \mathrm{~F}_{5 / 2} \\
5 \mathrm{~d}^{8}\left({ }^{3} \mathrm{P}\right) 6 \mathrm{~s} \text { a }{ }^{2} \mathrm{P}_{3 / 2}\end{array}$ & $\begin{array}{c}4078.94 \\
5784.62 \\
6324.91 \\
7106.61 \\
9877.54 \\
10578.68\end{array}$ & $\begin{array}{l}266.992 \\
279.735 \\
284.021 \\
290.481 \\
315.918 \\
323.076\end{array}$ & $\begin{array}{l}0.16 \\
0.24 \\
0.25 \\
0.14 \\
0.06 \\
0.11\end{array}$ & $\begin{array}{c}57.1(E) \\
85.7(D+) \\
89.3(D+) \\
50.0(E) \\
21.4(E) \\
39.3(E)\end{array}$ & & $\begin{array}{c}-1.21(E) \\
-1.00(D+) \\
-0.97(D+) \\
-1.20(E) \\
-1.49(E) \\
-1.21(E)\end{array}$ & & \\
\hline $4226^{\circ}{ }_{5 / 2}$ & $\begin{array}{c}42267.86 \\
\tau=23.3(13)\end{array}$ & $\begin{array}{c}5 \mathrm{~d}^{7} 6 \mathrm{~s}^{2} \text { a }{ }^{4} \mathrm{~F}_{3 / 2} \\
5 \mathrm{~d}^{7} 6 \mathrm{~s}^{2} \text { a }{ }^{4} \mathrm{~F}_{7 / 2} \\
5 \mathrm{~d}^{8}\left({ }^{3} \mathrm{~F}\right) 6 \mathrm{~s} \text { b }{ }^{4} \mathrm{~F}_{5 / 2}\end{array}$ & $\begin{array}{l}4078.94 \\
6323.91 \\
9877.54\end{array}$ & $\begin{array}{l}261.778 \\
278.129 \\
308.644\end{array}$ & $\begin{array}{l}0.50 \\
0.33 \\
0.09\end{array}$ & $\begin{array}{c}129(D+) \\
85.0(D+) \\
23.2(E)\end{array}$ & & $\begin{array}{c}-0.88(D+) \\
-1.01(D+) \\
-1.48(E)\end{array}$ & & \\
\hline $4227^{\circ} 9 / 2$ & $\begin{array}{c}42279.28 \\
\tau=74.0(27)\end{array}$ & $5 d^{8}\left({ }^{3} \mathrm{~F}\right) 6 s \mathrm{~b}{ }^{4} \mathrm{~F}_{9 / 2}$ & 2834.98 & 253.446 & 0.89 & $120(C+)$ & & $-0.94(C+)$ & & \\
\hline $4317^{\circ} 7 / 2$ & $\begin{array}{c}43176.15 \\
\tau=19.7(9)\end{array}$ & $\begin{array}{c}5 \mathrm{~d}^{7} 6 \mathrm{~s}^{2} \text { a }{ }^{4} \mathrm{~F}_{9 / 2} \\
5 \mathrm{~d}^{7} 6 \mathrm{~s}^{2} \text { a }{ }^{4} \mathrm{~F}_{5 / 2} \\
5 \mathrm{~d}^{7} 6 \mathrm{~s}^{2} \text { a }{ }^{4} \mathrm{~F}_{7 / 2} \\
5 \mathrm{~d}^{8}\left({ }^{3} \mathrm{~F}\right) 6 \mathrm{~s} \text { b }{ }^{4} \mathrm{~F}_{5 / 2} \\
5 \mathrm{~d}^{8}\left({ }^{3} \mathrm{~F}\right) 6 \mathrm{~s} \text { a }{ }^{2} \mathrm{~F}_{5 / 2} \\
5 \mathrm{~d}^{8}\left({ }^{3} \mathrm{~F}\right) 6 \mathrm{~s} \text { a }{ }^{2} \mathrm{~F}_{7 / 2}\end{array}$ & $\begin{array}{c}0.00 \\
5784.62 \\
6323.91 \\
9877.54 \\
12218.47 \\
13087.90\end{array}$ & $\begin{array}{l}231.538 \\
267.361 \\
271.274 \\
300.225 \\
322.929 \\
332.260\end{array}$ & $\begin{array}{l}0.09 \\
0.15 \\
0.14 \\
0.14 \\
0.18 \\
0.20\end{array}$ & $\begin{array}{c}36.5(E) \\
60.9(E) \\
56.8(E) \\
56.8(E) \\
73.1(E) \\
81.2(D+)\end{array}$ & & $\begin{array}{c}-1.53(E) \\
-1.19(E) \\
-1.20(E) \\
-1.11(E) \\
-0.94(E) \\
-0.87(D+)\end{array}$ & & \\
\hline
\end{tabular}


Table 4

(Continued)

\begin{tabular}{|c|c|c|c|c|c|c|c|c|c|c|}
\hline \multicolumn{2}{|c|}{ Upper Level $^{\mathrm{a}}$} & \multicolumn{2}{|c|}{ Lower Level $^{\mathrm{a}}$} & \multirow{3}{*}{$\lambda_{\text {air }}(\mathrm{nm})$} & \multirow{3}{*}{$\mathrm{BF}^{\mathrm{b}}$} & \multicolumn{2}{|c|}{$g A\left(10^{6} \mathrm{~s}^{-1}\right)$} & \multicolumn{3}{|c|}{$\log (g f)$} \\
\hline \multirow{2}{*}{ Assign. } & \multirow{2}{*}{$E\left(\mathrm{~cm}^{-1}\right)$ Lifetime (ns) } & \multirow{2}{*}{ Assign. } & \multirow{2}{*}{$E\left(\mathrm{~cm}^{-1}\right)$} & & & \multirow{2}{*}{ This work $^{\mathrm{b}}$} & \multirow{2}{*}{$\begin{array}{l}\text { Previous } \\
\text { Exp. }\end{array}$} & \multirow{2}{*}{ This work ${ }^{\mathrm{c}}$} & \multicolumn{2}{|c|}{ Previous } \\
\hline & & & & & & & & & Exp. & Calc. \\
\hline $4359^{\circ}{ }_{7 / 2}$ & $\begin{array}{c}43529.21 \\
\tau=56.2(56)\end{array}$ & $\begin{array}{c}5 d^{8}\left({ }^{3} F\right) 6 s \text { b }{ }^{4} F_{9 / 2} \\
5 d^{7} 6 s^{2} \text { a }{ }^{4} F_{5 / 2} \\
5 d^{8}\left({ }^{3} F\right) 6 s \text { b }{ }^{4} F_{7 / 2} \\
5 d^{8}\left({ }^{3} \mathrm{~F}\right) 6 s \text { b }{ }^{4} F_{5 / 2} \\
5 d^{8}\left({ }^{3} F\right) 6 s \text { a }{ }^{2} F_{7 / 2} \\
5 d^{7} 6 s^{2} \text { a }{ }^{2} G_{9 / 2}\end{array}$ & $\begin{array}{c}2834.98 \\
5784.62 \\
7106.61 \\
9877.54 \\
13087.90 \\
13939.80\end{array}$ & $\begin{array}{l}245.281 \\
264.418 \\
274.000 \\
296.520 \\
327.729 \\
337.144\end{array}$ & $\begin{array}{l}0.07 \\
0.10 \\
0.07 \\
0.05 \\
0.17 \\
0.09\end{array}$ & $\begin{array}{l}9.96(E) \\
14.2(E) \\
9.96(E) \\
7.12(E) \\
24.2(E) \\
12.8(E)\end{array}$ & & $\begin{array}{l}-2.04(E) \\
-1.82(E) \\
-1.95(E) \\
-2.02(E) \\
-1.41(E) \\
-1.66(E)\end{array}$ & & \\
\hline $4459^{\circ}{ }_{5 / 2}$ & $\begin{array}{c}44596.77 \\
\tau=17.5(14)\end{array}$ & $\begin{array}{c}5 d^{7} 6 s^{2} \text { a }{ }^{4} F_{3 / 2} \\
5 d^{8}\left({ }^{3} \mathrm{~F}\right) 6 s \text { b }{ }^{4} F_{5 / 2} \\
5 d^{8}\left({ }^{3} \mathrm{P}\right) 6 s \text { a }{ }^{4} \mathrm{P}_{5 / 2} \\
5 d^{8}\left({ }^{3} \mathrm{~F}\right) 6 s \text { a }{ }^{2} \mathrm{~F}_{7 / 2}\end{array}$ & $\begin{array}{c}4078.94 \\
9877.54 \\
12951.67 \\
13087.90\end{array}$ & $\begin{array}{l}246.730 \\
287.941 \\
315.914 \\
317.279\end{array}$ & $\begin{array}{l}0.31 \\
0.36 \\
0.09 \\
0.06\end{array}$ & $\begin{array}{c}106(D+) \\
123(D+) \\
30.9(E) \\
20.6(E)\end{array}$ & & $\begin{array}{c}-1.01(D+) \\
-0.82(D+) \\
-1.34(E) \\
-1.51(E)\end{array}$ & & \\
\hline $4464^{\circ}{ }_{7 / 2}$ & $\begin{array}{c}44642.67 \\
\tau=7.7(5)\end{array}$ & $\begin{array}{l}5 d^{8}\left({ }^{3} \mathrm{~F}\right) 6 \mathrm{~s} \text { b }{ }^{4} \mathrm{~F}_{9 / 2} \\
5 \mathrm{~d}^{8}\left({ }^{3} \mathrm{~F}\right) 6 \mathrm{~s} \text { a }{ }^{2} \mathrm{~F}_{5 / 2} \\
5 \mathrm{~d}^{8}\left({ }^{3} \mathrm{P}\right) 6 \mathrm{~s} \text { a }{ }^{4} \mathrm{P}_{5 / 2} \\
5 \mathrm{~d}^{8}\left({ }^{3} \mathrm{~F}\right) 6 \mathrm{~s} \text { a }{ }^{2} \mathrm{~F}_{7 / 2}\end{array}$ & $\begin{array}{c}2834.98 \\
12218.47 \\
12951.67 \\
13087.90\end{array}$ & $\begin{array}{l}239.117 \\
308.322 \\
315.456 \\
316.817\end{array}$ & $\begin{array}{l}0.51 \\
0.11 \\
0.07 \\
0.14\end{array}$ & $\begin{array}{c}530(D+) \\
114(E) \\
72.7(E) \\
145(E)\end{array}$ & & $\begin{array}{c}-0.34(D+) \\
-0.79(E) \\
-0.97(E) \\
-0.66(E)\end{array}$ & & \\
\hline $4465^{\circ} 9 / 2$ & $\begin{array}{c}44652.43 \\
\tau=12.2(8)\end{array}$ & $\begin{array}{c}5 d^{7} 6 s^{2} \text { a }{ }^{4} F_{9 / 2} \\
5 d^{8}\left({ }^{3} \mathrm{~F}\right) 6 s \text { b }{ }^{4} F_{9 / 2} \\
5 d^{7} 6 s^{2} \text { a }{ }^{4} F_{7 / 2} \\
5 d^{8}\left({ }^{3} \mathrm{~F}\right) 6 s \text { b }{ }^{4} F_{7 / 2} \\
5 d^{8}\left({ }^{3} \mathrm{~F}\right) 6 s \text { a }{ }^{2} \mathrm{~F}_{7 / 2}\end{array}$ & $\begin{array}{c}0.00 \\
2834.98 \\
6323.91 \\
7106.61 \\
13087.90\end{array}$ & $\begin{array}{l}223.882 \\
239.062 \\
260.824 \\
266.262 \\
316.719\end{array}$ & $\begin{array}{l}0.15 \\
0.31 \\
0.11 \\
0.34 \\
0.07\end{array}$ & $\begin{array}{c}123(E) \\
254(D+) \\
90.2(E) \\
279(D+) \\
57.4(E)\end{array}$ & & $\begin{array}{c}-1.03(E) \\
-0.66(D+) \\
-1.04(E) \\
-0.53(D+) \\
-1.06(E)\end{array}$ & & \\
\hline $4478_{3 / 2}^{\circ}$ & $\begin{array}{c}44785.44 \\
\tau=7.1(4)\end{array}$ & $\begin{array}{c}5 \mathrm{~d}^{8}\left({ }^{3} \mathrm{~F}\right) 6 \mathrm{~s} \text { b }{ }^{4} \mathrm{~F}_{5 / 2} \\
5 \mathrm{~d}^{8}\left({ }^{3} \mathrm{~F}\right) 6 \mathrm{~s} \text { a }{ }^{2} \mathrm{~F}_{5 / 2} \\
5 \mathrm{~d}^{8}\left({ }^{3} \mathrm{P}\right) 6 \mathrm{~s} \text { a }{ }^{4} \mathrm{P}_{5 / 2} \\
5 \mathrm{~d}^{7} 6 \mathrm{~s}^{2} \text { b }{ }^{4} \mathrm{P}_{5 / 2} \\
5 \mathrm{~d}^{8}\left({ }^{3} \mathrm{P}\right) 6 \mathrm{~s} \text { a }{ }^{4} \mathrm{P}_{1 / 2}\end{array}$ & $\begin{array}{c}9877.54 \\
12218.47 \\
12951.67 \\
16103.32 \\
16681.20\end{array}$ & $\begin{array}{l}286.384 \\
306.971 \\
314.041 \\
348.549 \\
355.716\end{array}$ & $\begin{array}{l}0.17 \\
0.27 \\
0.10 \\
0.14 \\
0.15\end{array}$ & $\begin{array}{c}95.8(E) \\
152(D+) \\
56.3(E) \\
78.9(E) \\
84.5(E)\end{array}$ & & $\begin{array}{c}-0.93(E) \\
-0.67(D+) \\
-1.08(E) \\
-0.84(E) \\
-0.79(E)\end{array}$ & & \\
\hline $4511^{\circ}{ }_{7 / 2}$ & $\begin{array}{c}45111.68 \\
\tau=17.9(8)\end{array}$ & $\begin{array}{c}5 d^{7} 6 s^{2} \text { a }{ }^{4} F_{5 / 2} \\
5 d^{8}\left({ }^{3} \mathrm{~F}\right) 6 s \text { b }{ }^{4} F_{7 / 2} \\
5 d^{8}\left({ }^{3} \mathrm{~F}\right) 6 s \text { b }{ }^{4} F_{5 / 2} \\
5 d^{8}\left({ }^{3} \mathrm{~F}\right) 6 s \text { a }{ }^{2} F_{5 / 2} \\
5 d^{8}\left({ }^{3} \mathrm{~F}\right) 6 s \text { a }{ }^{2} F_{7 / 2}\end{array}$ & $\begin{array}{c}5784.62 \\
7106.61 \\
9877.54 \\
12218.47 \\
13087.90\end{array}$ & $\begin{array}{l}254.202 \\
263.051 \\
283.739 \\
303.932 \\
312.180\end{array}$ & $\begin{array}{l}0.07 \\
0.08 \\
0.25 \\
0.42 \\
0.07\end{array}$ & $\begin{array}{c}31.3(E) \\
35.8(E) \\
112(D+) \\
188(D+) \\
31.3(E)\end{array}$ & & $\begin{array}{c}-1.51(E) \\
-1.43(E) \\
-0.87(D+) \\
-0.58(D+) \\
-1.34(E)\end{array}$ & & \\
\hline $4518^{\circ}{ }_{5 / 2}$ & $\begin{array}{c}45185.95 \\
\tau=10.4(3)\end{array}$ & $\begin{array}{l}5 d^{8}\left({ }^{3} \mathrm{~F}\right) 6 \mathrm{~s} \mathrm{~b}{ }^{4} \mathrm{~F}_{7 / 2} \\
5 \mathrm{~d}^{8}\left({ }^{3} \mathrm{~F}\right) 6 \mathrm{~s} \mathrm{~b}{ }^{4} \mathrm{~F}_{3 / 2} \\
5 \mathrm{~d}^{8}\left({ }^{3} \mathrm{~F}\right) 6 \mathrm{~s} \text { a }{ }^{2} \mathrm{~F}_{5 / 2} \\
5 \mathrm{~d}^{8}\left({ }^{3} \mathrm{~F}\right) 6 \mathrm{~s} \text { a }{ }^{2} \mathrm{~F}_{7 / 2}\end{array}$ & $\begin{array}{c}7106.61 \\
11831.09 \\
12218.47 \\
13087.90\end{array}$ & $\begin{array}{l}262.532 \\
299.719 \\
303.241 \\
311.455\end{array}$ & $\begin{array}{l}0.51 \\
0.07 \\
0.16 \\
0.07\end{array}$ & $\begin{array}{c}294(D+) \\
40.4(E) \\
92.3(E) \\
40.4(E)\end{array}$ & & $\begin{array}{l}-0.52(D+) \\
-1.26(E) \\
-0.89(E) \\
-1.23(E)\end{array}$ & & \\
\hline $4525^{\circ}{ }_{3 / 2}$ & $\begin{array}{c}45259.14 \\
\tau=16.8(5)\end{array}$ & $\begin{array}{c}5 \mathrm{~d}^{7} 6 \mathrm{~s}^{2} \text { a }{ }^{4} \mathrm{~F}_{3 / 2} \\
5 \mathrm{~d}^{7} 6 \mathrm{~s}^{2} \text { a }{ }^{4} \mathrm{~F}_{5 / 2} \\
5 \mathrm{~d}^{8}\left({ }^{3} \mathrm{~F}\right) 6 \mathrm{~s} \mathrm{~b}{ }^{4} \mathrm{~F}_{5 / 2} \\
5 \mathrm{~d}^{8}\left({ }^{3} \mathrm{~F}\right) 6 \mathrm{~s} \text { b }{ }^{4} \mathrm{~F}_{3 / 2}\end{array}$ & $\begin{array}{c}4078.94 \\
5784.62 \\
9877.54 \\
11831.09\end{array}$ & $\begin{array}{l}242.761 \\
253.252 \\
282.550 \\
299.063\end{array}$ & $\begin{array}{l}0.16 \\
0.23 \\
0.08 \\
0.34\end{array}$ & $\begin{array}{c}38.1(E) \\
54.8(D+) \\
19.0(E) \\
80.9(D+)\end{array}$ & & $\begin{array}{c}-1.47(E) \\
-1.28(D+) \\
-1.64(E) \\
-0.96(D+)\end{array}$ & & \\
\hline
\end{tabular}


Table 4

(Continued)

\begin{tabular}{|c|c|c|c|c|c|c|c|c|c|c|}
\hline \multicolumn{2}{|c|}{ Upper Level $^{\mathrm{a}}$} & \multicolumn{2}{|c|}{ Lower Level $^{\mathrm{a}}$} & \multirow{3}{*}{$\lambda_{\text {air }}(\mathrm{nm})$} & \multirow[t]{3}{*}{$\mathrm{BF}^{\mathrm{b}}$} & \multicolumn{2}{|c|}{$g A\left(10^{6} \mathrm{~s}^{-1}\right)$} & \multicolumn{3}{|c|}{$\log (g f)$} \\
\hline \multirow{2}{*}{ Assign. } & \multirow{2}{*}{$E\left(\mathrm{~cm}^{-1}\right)$ Lifetime (ns) } & \multirow{2}{*}{ Assign. } & \multirow{2}{*}{$E\left(\mathrm{~cm}^{-1}\right)$} & & & \multirow{2}{*}{ This work ${ }^{\mathrm{b}}$} & \multirow{2}{*}{$\begin{array}{l}\text { Previous } \\
\text { Exp. }\end{array}$} & \multirow{2}{*}{ This work ${ }^{c}$} & \multicolumn{2}{|c|}{ Previous } \\
\hline & & & & & & & & & Exp. & Calc. \\
\hline $4557^{\circ}{ }_{5 / 2}$ & $\begin{array}{c}45570.89 \\
\tau=13.1(4)\end{array}$ & $\begin{array}{c}5 d^{7} 6 s^{2} \text { a }{ }^{4} F_{3 / 2} \\
5 d^{7} 6 s^{2} \text { a }{ }^{4} F_{5 / 2} \\
5 d^{8}\left({ }^{3} \mathrm{~F}\right) 6 s \text { b }{ }^{4} F_{5 / 2}\end{array}$ & $\begin{array}{l}4078.94 \\
5784.62 \\
9877.54\end{array}$ & $\begin{array}{l}240.938 \\
251.267 \\
280.081\end{array}$ & $\begin{array}{l}0.63 \\
0.06 \\
0.15\end{array}$ & $\begin{array}{l}289(C) \\
27.5(E) \\
68.7(E)\end{array}$ & & $\begin{array}{l}-0.61(C) \\
-1.59(E) \\
-1.10(E)\end{array}$ & & \\
\hline $4589^{\circ} 7 / 2$ & $\begin{array}{c}45895.85 \\
\tau=7.6(2)\end{array}$ & $\begin{array}{c}5 d^{7} 6 s^{2} \text { a }{ }^{4} F_{9 / 2} \\
5 d^{8}\left({ }^{3} \mathrm{~F}\right) 6 s \text { b }{ }^{4} \mathrm{~F}_{7 / 2} \\
5 d^{8}\left({ }^{3} \mathrm{~F}\right) 6 s \text { a }{ }^{2} \mathrm{~F}_{7 / 2} \\
5 \mathrm{~d}^{7} 6 \mathrm{~s}^{2} \text { a }{ }^{2} \mathrm{G}_{9 / 2}\end{array}$ & $\begin{array}{c}0.00 \\
7106.61 \\
13087.90 \\
13939.80\end{array}$ & $\begin{array}{l}217.817 \\
257.726 \\
304.715 \\
312.839\end{array}$ & $\begin{array}{l}0.23 \\
0.27 \\
0.28 \\
0.11\end{array}$ & $\begin{array}{c}242(D+) \\
284(D+) \\
295(D+) \\
116(E)\end{array}$ & & $\begin{array}{c}-0.76(D+) \\
-0.55(D+) \\
-0.39(D+) \\
-0.77(E)\end{array}$ & & \\
\hline $4622^{\circ} 9 / 2$ & $\begin{array}{c}46220.32 \\
\tau=11.4(6)\end{array}$ & $\begin{array}{c}5 d^{7} 6 s^{2} \text { a }{ }^{4} F_{9 / 2} \\
5 d^{8}\left({ }^{3} \mathrm{~F}\right) 6 s \text { b }{ }^{4} \mathrm{~F}_{9 / 2} \\
5 d^{7} 6 s^{2} \text { a }{ }^{4} F_{7 / 2} \\
5 d^{8}\left({ }^{3} \mathrm{~F}\right) 6 s \text { a }{ }^{2} \mathrm{~F}_{7 / 2}\end{array}$ & $\begin{array}{c}0.00 \\
2834.98 \\
6323.91 \\
13087.90\end{array}$ & $\begin{array}{l}216.287 \\
230.422 \\
250.574 \\
301.731\end{array}$ & $\begin{array}{l}0.36 \\
0.28 \\
0.05 \\
0.26\end{array}$ & $\begin{array}{c}316(D+) \\
246(D+) \\
43.9(E) \\
228(D+)\end{array}$ & & $\begin{array}{c}-0.65(D+) \\
-0.71(D+) \\
-1.38(E) \\
-0.51(D+)\end{array}$ & & \\
\hline $4637^{\circ} 9 / 2$ & $\begin{array}{c}46371.64 \\
\tau=22.6(8)\end{array}$ & $\begin{array}{c}5 d^{7} 6 s^{2} \text { a }{ }^{4} F_{9 / 2} \\
5 d^{8}\left({ }^{3} \mathrm{~F}\right) 6 s \text { b }{ }^{4} \mathrm{~F}_{9 / 2} \\
5 d^{7} 6 s^{2} \text { a }{ }^{4} \mathrm{~F}_{7 / 2} \\
5 d^{8}\left({ }^{3} \mathrm{~F}\right) 6 s \text { b }{ }^{4} \mathrm{~F}_{7 / 2} \\
5 \mathrm{~d}^{8}\left({ }^{3} \mathrm{~F}\right) 6 \mathrm{~s} \text { a }{ }^{2} \mathrm{~F}_{7 / 2}\end{array}$ & $\begin{array}{c}0.00 \\
2834.98 \\
6323.91 \\
7106.61 \\
13087.90\end{array}$ & $\begin{array}{l}215.581 \\
229.620 \\
249.627 \\
254.604 \\
300.359\end{array}$ & $\begin{array}{l}0.45 \\
0.08 \\
0.14 \\
0.20 \\
0.09\end{array}$ & $\begin{array}{c}199(D+) \\
35.4(E) \\
61.9(E) \\
88.5(D+) \\
39.8(E)\end{array}$ & & $\begin{array}{c}-0.86(D+) \\
-1.55(E) \\
-1.24(E) \\
-1.06(D+) \\
-1.27(E)\end{array}$ & & \\
\hline $4716^{\circ} 7 / 2$ & $\begin{array}{c}47165.12 \\
\tau=14.3(8)\end{array}$ & $\begin{array}{c}5 d^{7} 6 s^{2} \text { a }{ }^{4} F_{5 / 2} \\
5 d^{7} 6 s^{2} \text { a }{ }^{4} F_{7 / 2} \\
5 d^{8}\left({ }^{3} \mathrm{~F}\right) 6 s \text { b }{ }^{4} \mathrm{~F}_{7 / 2} \\
5 d^{8}\left({ }^{3} \mathrm{~F}\right) 6 s \text { a }{ }^{2} \mathrm{~F}_{5 / 2} \\
5 \mathrm{~d}^{8}\left({ }^{3} \mathrm{~F}\right) 6 \mathrm{~s} \text { a }{ }^{2} \mathrm{~F}_{7 / 2}\end{array}$ & $\begin{array}{c}5784.62 \\
6323.91 \\
7106.61 \\
12218.47 \\
13087.90\end{array}$ & $\begin{array}{l}241.587 \\
244.777 \\
249.560 \\
286.066 \\
293.365\end{array}$ & $\begin{array}{l}0.06 \\
0.24 \\
0.11 \\
0.36 \\
0.07\end{array}$ & $\begin{array}{c}33.6(E) \\
134(D+) \\
61.5(E) \\
201(D+) \\
39.2(E)\end{array}$ & & $\begin{array}{c}-1.53(E) \\
-0.92(D+) \\
-1.24(E) \\
-0.61(D+) \\
-1.30(E)\end{array}$ & & \\
\hline $4720_{9 / 2}^{\circ}$ & $\begin{array}{c}47205.57 \\
\tau=31.7(11)\end{array}$ & $\begin{array}{c}5 d^{7} 6 s^{2} \text { a }{ }^{4} F_{9 / 2} \\
5 d^{8}\left({ }^{3} F\right) 6 s \text { b }{ }^{4} F_{9 / 2} \\
5 d^{7} 6 s^{2} \text { a }{ }^{4} F_{7 / 2} \\
5 d^{8}\left({ }^{3} F\right) 6 s \text { b }{ }^{4} F_{7 / 2} \\
5 d^{7} 6 s^{2} \text { a }{ }^{2} G_{9 / 2}\end{array}$ & $\begin{array}{c}0.00 \\
2834.98 \\
6323.91 \\
7106.61 \\
13939.80\end{array}$ & $\begin{array}{l}211.772 \\
225.305 \\
244.534 \\
249.308 \\
300.521\end{array}$ & $\begin{array}{l}0.09 \\
0.05 \\
0.12 \\
0.64 \\
0.06\end{array}$ & $\begin{array}{l}28.4(E) \\
15.8(E) \\
37.9(E) \\
202(E) \\
18.9(E)\end{array}$ & & $\begin{array}{l}-1.72(E) \\
-1.92(E) \\
-1.47(E) \\
-0.73(E) \\
-1.59(E)\end{array}$ & & \\
\hline $4754^{\circ} 7 / 2$ & $\begin{array}{c}47548.69 \\
\tau=21.7(7)\end{array}$ & $\begin{array}{c}5 d^{7} 6 s^{2} a^{4} F_{9 / 2} \\
5 d^{8}\left({ }^{3} \mathrm{~F}\right) 6 s \text { b }{ }^{4} F_{9 / 2} \\
5 d^{8}\left({ }^{3} \mathrm{~F}\right) 6 s \text { b }{ }^{4} \mathrm{~F}_{5 / 2} \\
5 d^{8}\left({ }^{3} \mathrm{~F}\right) 6 s \text { a }{ }^{2} \mathrm{~F}_{5 / 2} \\
5 d^{7} 6 s^{2} \text { b }{ }^{4} \mathrm{P}_{5 / 2}\end{array}$ & $\begin{array}{c}0.00 \\
2834.98 \\
9877.54 \\
12218.47 \\
16103.32\end{array}$ & $\begin{array}{l}210.244 \\
223.576 \\
265.376 \\
282.961 \\
317.920\end{array}$ & $\begin{array}{c}0.17 \\
0.23 \\
0.07^{*} \\
0.13 \\
0.19\end{array}$ & $\begin{array}{c}62.7(E) \\
84.8(D+) \\
25.8(E) \\
47.9(E) \\
70.0(E)\end{array}$ & & $\begin{array}{l}-1.38(E) \\
-1.20(D+) \\
-1.56(E) \\
-1.24(E) \\
-0.97(E)\end{array}$ & & \\
\hline $4785^{\circ}{ }_{11 / 2}$ & $\begin{array}{c}47858.47 \\
\tau=3.7(3)\end{array}$ & $\begin{array}{c}5 d^{7} 6 s^{2} \text { a }{ }^{4} F_{9 / 2} \\
5 d^{8}\left({ }^{3} \mathrm{~F}\right) 6 s \text { b }{ }^{4} F_{9 / 2}\end{array}$ & $\begin{array}{c}0.00 \\
2834.98\end{array}$ & $\begin{array}{l}208.883 \\
222.037\end{array}$ & $\begin{array}{l}0.89 \\
0.11\end{array}$ & $\begin{array}{c}2886(C+) \\
357(E)\end{array}$ & & $\begin{array}{l}0.28(C+) \\
-0.58(E)\end{array}$ & & \\
\hline $4820^{\circ}{ }_{5 / 2}$ & $\begin{array}{c}48206.57 \\
\tau=8.3(7)\end{array}$ & $\begin{array}{c}5 d^{7} 6 s^{2} \text { a }{ }^{4} \mathrm{~F}_{3 / 2} \\
5 d^{7} 6 s^{2} \text { a }{ }^{4} \mathrm{~F}_{7 / 2} \\
5 \mathrm{~d}^{8}\left({ }^{3} \mathrm{~F}\right) 6 \mathrm{~s} \mathrm{~b}{ }^{4} \mathrm{~F}_{5 / 2} \\
5 \mathrm{~d}^{8}\left({ }^{3} \mathrm{P}\right) 6 \mathrm{~s} \text { a }{ }^{2} \mathrm{P}_{3 / 2}\end{array}$ & $\begin{array}{c}4078.94 \\
6323.91 \\
9877.54 \\
10578.68\end{array}$ & $\begin{array}{l}226.545 \\
238.689 \\
260.821 \\
265.681\end{array}$ & $\begin{array}{l}0.16 \\
0.30 \\
0.15 \\
0.16\end{array}$ & $\begin{array}{c}116(E) \\
217(D+) \\
108(E) \\
116(E)\end{array}$ & & $\begin{array}{l}-1.05(E) \\
-0.73(D+) \\
-0.96(E) \\
-0.91(E)\end{array}$ & & \\
\hline
\end{tabular}


Table 4

(Continued)

\begin{tabular}{|c|c|c|c|c|c|c|c|c|c|c|}
\hline \multicolumn{2}{|c|}{ Upper Level $^{\mathrm{a}}$} & \multicolumn{2}{|c|}{ Lower Level $^{\mathrm{a}}$} & \multirow{3}{*}{$\lambda_{\text {air }}(\mathrm{nm})$} & \multirow[t]{3}{*}{$\mathrm{BF}^{\mathrm{b}}$} & \multicolumn{2}{|c|}{$g A\left(10^{6} \mathrm{~s}^{-1}\right)$} & \multicolumn{3}{|c|}{$\log (g f)$} \\
\hline \multirow[t]{2}{*}{ Assign. } & \multirow{2}{*}{$E\left(\mathrm{~cm}^{-1}\right)$ Lifetime $(\mathrm{ns})$} & \multirow[t]{2}{*}{ Assign. } & \multirow[t]{2}{*}{$E\left(\mathrm{~cm}^{-1}\right)$} & & & \multirow[t]{2}{*}{ This work ${ }^{\mathrm{b}}$} & \multirow{2}{*}{$\begin{array}{l}\text { Previous } \\
\text { Exp. }\end{array}$} & \multirow[t]{2}{*}{ This work ${ }^{\mathrm{c}}$} & \multicolumn{2}{|c|}{ Previous } \\
\hline & & & & & & & & & Exp. & Calc. \\
\hline & & $5 d^{8}\left({ }^{3} \mathrm{P}\right) 6 \mathrm{~s}$ a ${ }^{4} \mathrm{P}_{5 / 2}$ & 12951.67 & 283.566 & $0.09^{*}$ & $65.1(E)$ & & $-1.11(E)$ & & \\
\hline & & $5 d^{7} 6 s^{2} b{ }^{4} P_{3 / 2}$ & 18547.04 & 337.063 & 0.05 & $36.1(E)$ & & $-1.21(E)$ & & \\
\hline \multirow[t]{3}{*}{$4844^{\circ}{ }_{3 / 2}$} & 48440.83 & $5 \mathrm{~d}^{8}\left({ }^{3} \mathrm{P}\right) 6 \mathrm{~s}$ a ${ }^{2} \mathrm{P}_{3 / 2}$ & 10578.68 & 264.037 & $0.07^{*}$ & $45.2(E)$ & & $-1.32(E)$ & & \\
\hline & $\tau=6.2(2)$ & $5 d^{8}\left({ }^{3} F\right) 6 s$ b ${ }^{4} F_{3 / 2}$ & 11831.09 & 273.070 & 0.62 & $400(C)$ & & $-0.35(C)$ & & \\
\hline & & $5 \mathrm{~d}^{8}\left({ }^{3} \mathrm{P}\right) 6 \mathrm{~s}$ a ${ }^{2} \mathrm{P}_{1 / 2}$ & 12505.68 & 278.197 & 0.10 & $64.5(D)$ & & $-1.13(D)$ & & \\
\hline \multirow{4}{*}{$4844^{\circ}{ }_{7 / 2}$} & 48448.65 & $5 d^{7} 6 s^{2}$ a ${ }^{4} F_{5 / 2}$ & 5784.62 & 234.317 & 0.09 & $84.7(E)$ & & $-1.16(E)$ & & \\
\hline & $\tau=8.5(3)$ & $5 d^{7} 6 s^{2}$ a ${ }^{4} F_{7 / 2}$ & 6323.91 & 237.318 & 0.55 & $517(D+)$ & & $-0.36(D+)$ & & \\
\hline & & $5 d^{8}\left({ }^{3} F\right) 6 s$ b ${ }^{4} F_{7 / 2}$ & 7106.61 & 241.812 & 0.14 & $132(E)$ & & $-0.94(E)$ & & \\
\hline & & $5 d^{8}\left({ }^{3} \mathrm{~F}\right) 6 \mathrm{~s}$ a ${ }^{2} \mathrm{~F}_{5 / 2}$ & 12218.47 & 275.931 & 0.10 & $94.1(E)$ & & $-0.97(E)$ & & \\
\hline \multirow[t]{5}{*}{$4862^{\circ} 7 / 2$} & 48629.22 & $5 d^{7} 6 s^{2}$ a ${ }^{4} F_{9 / 2}$ & 0.00 & 205.572 & 0.06 & $76.2(E)$ & & $-1.32(E)$ & & \\
\hline & $\tau=6.3(3)$ & $5 \mathrm{~d}^{8}\left({ }^{3} \mathrm{~F}\right) 6 \mathrm{~s} \mathrm{~b}{ }^{4} \mathrm{~F}_{9 / 2}$ & 2834.98 & 218.300 & 0.45 & $571(D+)$ & & $-0.39(D+)$ & & \\
\hline & & $5 d^{7} 6 s^{2}$ a ${ }^{4} F_{5 / 2}$ & 5784.62 & 233.330 & 0.24 & $305(D+)$ & & $-0.60(D+)$ & & \\
\hline & & $5 \mathrm{~d}^{8}\left({ }^{3} \mathrm{~F}\right) 6 \mathrm{~s} \mathrm{~b}{ }^{4} \mathrm{~F}_{7 / 2}$ & 7106.61 & 240.760 & 0.09 & $114(E)$ & & $-1.00(E)$ & & \\
\hline & & $5 d^{8}\left({ }^{3} F\right) 6 s b{ }^{4} F_{5 / 2}$ & 9877.54 & 257.976 & 0.05 & $63.5(E)$ & & $-1.20(E)$ & & \\
\hline \multirow[t]{7}{*}{$4977^{\circ}{ }_{5 / 2}$} & 49779.37 & $5 d^{7} 6 s^{2}$ a ${ }^{4} F_{3 / 2}$ & 4078.94 & 218.748 & 0.15 & $81.8(E)$ & & $-1.23(E)$ & & \\
\hline & $\tau=11.0(6)$ & $5 d^{7} 6 s^{2}$ a ${ }^{4} F_{7 / 2}$ & 6323.91 & 230.050 & 0.10 & $54.5(E)$ & & $-1.36(E)$ & & \\
\hline & & $5 d^{8}\left({ }^{3} F\right) 6 s b^{4} F_{7 / 2}$ & 7106.61 & 234.270 & 0.18 & $98.2(E)$ & & $-1.09(E)$ & & \\
\hline & & $5 d^{8}\left({ }^{3} \mathrm{P}\right) 6 \mathrm{~s}$ a ${ }^{2} \mathrm{P}_{3 / 2}$ & 10578.68 & 255.021 & 0.12 & $65.5(E)$ & & $-1.20(E)$ & & \\
\hline & & $5 \mathrm{~d}^{8}\left({ }^{3} \mathrm{~F}\right) 6 \mathrm{~s} \mathrm{a}^{2} \mathrm{~F}_{7 / 2}$ & 13087.90 & 272.462 & 0.16 & $87.3(E)$ & & $-1.01(E)$ & & \\
\hline & & $5 d^{8}\left({ }^{3} \mathrm{P}\right) 6 \mathrm{~s}$ a ${ }^{4} \mathrm{P}_{3 / 2}$ & 16565.35 & 300.990 & 0.08 & $43.6(E)$ & & $-1.23(E)$ & & \\
\hline & & $5 d^{7} 6 s^{2} b{ }^{4} P_{3 / 2}$ & 18547.04 & 320.089 & 0.08 & $43.6(E)$ & & $-1.17(E)$ & & \\
\hline \multirow[t]{4}{*}{$4982^{\circ} 7 / 2$} & 49823.54 & $5 d^{7} 6 s^{2}$ a ${ }^{4} F_{5 / 2}$ & 5784.62 & 227.002 & 0.29 & $143(D+)$ & & $-0.96(D+)$ & & \\
\hline & $\tau=16.2(5)$ & $5 d^{7} 6 s^{2}$ a ${ }^{4} F_{7 / 2}$ & 6323.91 & 229.816 & 0.31 & $153(D+)$ & & $-0.92(D+)$ & & \\
\hline & & $5 d^{8}\left({ }^{3} F\right) 6 s b{ }^{4} F_{5 / 2}$ & 9877.54 & 250.263 & 0.15 & $74.1(E)$ & & $-1.16(E)$ & & \\
\hline & & $5 d^{8}\left({ }^{1} D\right) 6 s a^{2} D_{5 / 2}$ & 19060.62 & 324.973 & 0.09 & $44.4(E)$ & & $-1.15(E)$ & & \\
\hline
\end{tabular}

Notes.

${ }^{\mathrm{a}}$ Kramida et al. (2018).

b Calculated branching fractions with * are characterized by cancellation factors smaller than 0.01 (see text).

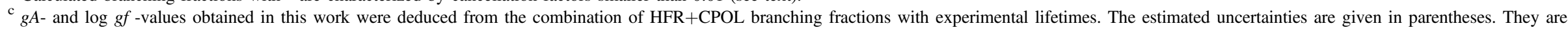
indicated by the same letter coding one used in the NIST database (Kramida et al. 2018), i.e., $B+(\leqslant 7 \%), B(\leqslant 10 \%), C+(\leqslant 18 \%), C(\leqslant 25 \%), D+(\leqslant 40 \%), D(\leqslant 50 \%)$, and $E(>50 \%)$ (see the text).

d Xu et al. (2007).

${ }^{\mathrm{e}}$ Gough et al. (1983). 


\section{ORCID iDs}

Zhenwen Dai (1) https://orcid.org/0000-0002-9040-2315

\section{References}

Colarusso, P., Lebeault Dorget, M. A., \& Simard, B. 1997, PhRvA, 55, 1526 Cowan, R. D. 1981, The Theory of Atomic Structure and Spectra (Berkeley, CA: Univ. California Press)

Den Hartog, E. A., Bilty, K. A., \& Lawler, J. E. 2011, JPhB, 44, 055001

Feng, Y. Y., Jiang, L. Y., Wang, Q., et al. 2011, MNRAS, 418, 998

Fraga, S., Karwowski, J., \& Saxena, K. M. S. 1976, Handbook of Atomic Data (Amsterdam: Elsevier)

Gough, D. S., Hannaford, P., \& Lowe, R. M. 1983, JPhB, 16, 785
Ivarsson, S., Andersen, J., Nordström, B., et al. 2003, A\&A, 409, 1141

Ivarsson, S., Wahlgren, G. M., Dai, Z., Lundberg, H., \& Leckrone, D. S. 2004, A\&A, 425, 353

Kramida, A., Ralchenko, Yu., Reader, J. \& NIST ASD Team 2018 NIST Atomic Spectra Database (ver. 5.5.6; Gaithersburg, MD: NIST), https://www.nist.gov/pml/atomic-spectra-database

Li, Z. S., Norin, J., Persson, A., Wahlström, C. G., \& Svanberg, S. 1999, PhRvA, 60, 198

Quinet, P., Palmeri, P., Biémont, E., et al. 1999, MNRAS, 307, 934

Quinet, P., Palmeri, P., Biémont, E., et al. 2002, JA11C, 344, 255

Ramanujam, P. S., \& Andersen, T. 1978, APJ, 226, 1171

Tian, Y. S., Wang, X. H., Yu, Q., et al. 2016, MNRAS, 457, 1393

Xu, H. L., Svanberg, S., Quinet, P., Palmeri, P., \& Biémont, E. 2007, JQSRT, 104,52 